\title{
Additions to the cercosporoid fungi from the Brazilian Cerrado: 1. New species on hosts belonging in family Fabaceae, and reallocations of four Stenella species into Zasmidium
}

\section{Antonio Hernández-Gutiérrez ${ }^{1}$, Zuleide Martins Chaves ${ }^{2}$, Denise Dornelo-Silva $^{2} \&$ José Carmine Dianese ${ }^{2 *}$}

${ }^{1}$ Departamento de Fitopatologia, Universidade Federal do Pará, Rua Augusto Corrêa 01, Campus do Guamá, 66071-110 Belém, Pará, Brazil

${ }^{2}$ Departamento de Fitopatologia, Universidade de Brasília, 70910-900, Brasília, Distrito Federal, Brazil

Received 25 November 2015 / Accepted 8 December 2015 / Published 18 December 2015

Hernández-Gutiérrez, A., Chaves, Z.M., Dornelo-Silva, D. \& Dianese, J.C. 2015. Additions to the cercosporoid fungi from the Brazilian Cerrado: 1. New species on hosts belonging in family Fabaceae, and reallocations of four Stenella species into Zasmidium. - Mycobiota 5: 33-64. doi: 10.12664/ mycobiota.2015.05.06

Abstract. Nine new species of cercosporoid fungi were found on leaves of plants in family Fabaceae from the Brazilian Cerrado: Asperisporium galactiae on Galactia peduncularis, Sirosporium sclerolobii and Zasmidium sclerolobii on Sclerolobium paniculatum, Pseudocercospora acosmii-subelegantis on Acosmium subelegans, and Pseudocercospora stryphnodendri on Stryphnodendron adstringens. In addition, four other Pseudocercospora species were found on Cassia s. lat., including Chamaecrista and Senna: Pseudocercospora aquae-emendadasensis on Chamaecrista orbiculata; Pseudocercospora sennae, Pseudocercospora sennae-rugosae, and Pseudocercospora subcuticularis on Senna rugosa. Descriptions, illustrations, and discussions of the new taxa are supplemented by keys to Pseudocercospora species on hosts belonging to Cassia s. lat., and to Zasmidium species on Fabaceae.

Key words: Asperisporium, foliicolous fungi, fungal taxonomy, leaf spots, Neotropical mycodiversity, Pseudocercospora, Sirosporium

\footnotetext{
*Corresponding author: e-mail: jcarmine@gmail.com
} 


\section{Introduction}

A survey accomplished in 2008 indicated that the Fabaceae (including subfamilies Caesalpinoideae, Faboideae, and Mimosoideae), known as the largest family of flowering plants in the world, encompasses in the Cerrado 108 genera, 1,174 species, and 262 varieties and subspecies. The Fabaceae corresponds to $9.5 \%$ of the total number $(>12,000$ species) of higher plant species in the Cerrado, including angiosperms, gymnosperms, and pteridophytes (Mendonça et al. 2008). However, studies on legume-associated cercosporoid fungi from the Cerrado are very scarce and confined to species described by Viégas (1945), included in Chupp (1954), some of them revised by Inácio et al. (1996) and Crous et al. (1997), and a few by Batista et al. (1960). Starting in 1994 (Medeiros \& Dianese 1994), a sequence of papers was published describing new species of cercosporoid fungi from the Cerrado. In these publications, a new species belonging in Parastenella, four in Cercospora, 22 in Passalora, 31 in Pseudocercospora, and five in Stenella were described (Dianese \& Câmara 1994; Inácio \& Dianese 1998, 1999, 2006; Furlanetto \& Dianese 1999; DorneloSilva \& Dianese 2003; Dornelo-Silva et al. 2007; Dianese et al. 2008; Hernández-Gutiérrez \& Dianese 2008, 2009, 2014a, b; Hernández-Gutiérrez et al. 2014). Four of the species previously assigned to Stenella are now reallocated to Zasmidium. On fabaceous hosts only eight new species distributed among the genera Cercospora, Passalora, and Pseudocercospora have recently been described (Hernández-Gutiérrez \& Dianese 2009). The present paper deals with new species of Asperisporium, Pseudocercospora, Sirosporium, and Zasmidium on plants belonging to Fabaceae, and reallocations of Stenella species from the Cerrado into Zasmidium.

\section{Material and methods}

Leaves showing necrotic spots of different shades of gray/brown were collected and dried in plant presses, before processing and depositing them in Herbarium UB Mycological Collection. Colonies on the leaf spots were initially examined using a Zeiss (Discovery v.8) stereomicroscope, to look for synnematous or fasciculate conidiophores. Portions of the fungal colonies were removed from the leaves and mounted on slides containing lactoglycerol. Then, light microscopic observations, measurements, and drawings were made using a Zeiss microscope, coupled to a camera lucida. The drawings show details of stromata, condidiophores, conidiogenous cells, and conidia of each specimen studied.

\section{Taxonomy}

Asperisporium galactiae A. Hern.-Gut., Z.M. Chaves \& Dianese, sp. nov.

Fig. 1 MycoBank MB 814798

Holotype: BRAZIL. Mato Grosso: Cuiabá, Chapada dos Guimarães National Park, Morro São Jerônimo, on leaves of Galactia peduncularis (Benth.) Taub. (FabaceaelFaboideae), 11 Apr 1996, J.C. Dianese (UB - Mycol Col. 11332). 
Etymology: galactiae, derived from the host plant genus Galactia.

Diagnosis: Differs from the type species, Asperisporium caricae (Speg.) Maubl., in having sublunate to fusoid conidia (vs. short-cylindrical in A. caricae).

Description: Lesions $0.5-2 \mathrm{~mm}$ diam., amphigenous, sparse, circular or irregular, brown, without defined margin and halo. Colonies brown, caespitose, mostly hypophyllous, sometimes epiphyllous. Mycelium internal. Stromata well-developed, textura globosa, erumpent, light brown, 23-50 $\mu \mathrm{m}$ diam. Conidiophores 0-2-septate, sometimes reduced to a simple conidiogenous cell, in compact sporodochia, straight, lageniform, cylindrical, ampulliform when immature, 17-38 $\mu \mathrm{m}$ long, $6-8 \mu \mathrm{m}$ broad in the widest part, light brown, smooth, thinwalled. Conidiogenous cells terminal, integrated, polyblastic, sympodial, with prominent thick, dark conidiogenous loci, $1.5-2.5 \mu \mathrm{m}$ wide. Conidia solitary, sublunate to fusoid, obconically truncate to truncate at the base, with thick, dark hilum, $1-2 \mu \mathrm{m}$ wide, apex rounded to broadly rounded, 33-40 $\mu \mathrm{m}$ long, 5-8 $\mu \mathrm{m}$ diam. in the widest part, 2-3 $\mu \mathrm{m}$ at the base, 1-3 $\mu \mathrm{m}$ near the apex, $0-5$-septate, dark brown, strongly verrucose when mature.

Comment: Crous \& Braun (2003) and Braun et al. (2013) considered Asperisporium close to Passalora, but differing by possessing strongly verrucose conidia. However, sequencing of the ITS region and nLSU of the Asperisporium type species, A. caricae, showed that it clustered within the Mycosphaerellaceae clade close to several Passalora species, but excluding the type species of the latter genus (Minnis et al. 2011). Thus, Braun et al. (2013) also admitted that further molecular work is needed to better clarify the situation of the polyphyletic genus Passalora. They also suggested that species with lightly verruculose conidia, as for instance $A$. sequoiae (Ellis \& Everh.) B. Sutton \& Hodges and A. juniperinum (Georgescu $\&$ Badea) B. Sutton $\&$ Hodges, clustering close to Passalora species and further away from the Asperisporium type species (Minnis et al. 2011), should be assigned to Passalora s. lat. Therefore, it is currently advisable to assign phylogenetically unresolved species that morphologically agree with the type of Asperisporium to the latter genus, at least tentatively.

There are 22 species of Asperisporium described and illustrated in the literature (http:// indexfungorum.org, September 2015). Most of the species agree morphologically well with the type species, A. caricae (Ellis 1971, 1976). Recently, A. caricicola Crous \& C. Nakash., a species morphologically indistinguishable from the type species has been described. The authors based the introduction of the new species on minor differences in ITS and LSU sequences; and the data was generated by comparing just one isolate of $A$. caricae with one of that considered as new species (Crous et al. 2015). When one takes into account that morphology reflects the entire genome of a fungus, it is difficult to accept a new species just based on minor differences in sequences whose phylogenetic meaning is hardly appraisable. In addition, it is more than questionable to base such a critical species on an unacceptably small sampling. Carica papaya is a species naturally distributed in coastal regions of tropical America, but $A$. caricicola was based on a single collection from cultivated papaya on Fiji, Oceania, which raised the question if, indeed, two morphologically indistinguishable Asperisporium have been evolved on this host or if the sequences just reflect intraspecific genetic variation. 
This question can barely be answered just based on two sequences. In any case, the precipitate introduction of $A$. caricicola is little helpful and more confusing to phytopathologists.

Asperisporium galactiae forms dark, subcuticular or subepidermal stromata, with textura globosa. This characteristic is also present in A. moringae (Ellis 1976); all other species of Asperisporium have stromata with textura angularis. Conidiophores of most species are cylindrical or occasionally obclavate, but conidiophores of $A$. galactiae range from ampulliform when young, to lageniform or even cylindrical when mature, and they are 0-1-septate. The conidia of Asperisporium species are in general uniformly 0-1(-2)-septa, except for A. mikaniae (Ellis \& Everh.) R.W. Barreto (Barreto \& Evans 1995) that may show conidia up to 5 -septate, as is the case in $A$. galactiae.

The conidia of $A$. galactiae are well characterized by being slightly curved (sublunate), wider at the middle, and strongly verrucose, but not verruculose at the base, with a more or less prominent dark hilum. The conidiogenous cells of $A$. galactiae are very characteristic by having prominent, thick, dark colored conidiogenous loci, appearing as broad short denticles, giving a peculiar shape to the conidiogenous cell apex.

Two species assigned to Asperisporium are known to be parasitic on hosts of the Fabaceae, viz. A. cassiae (Syd.) Deighton on Senna multiglandulosa (Jacq.) H.S. Irwin \& Barneby (= Cassia tomentosa L. f.), and A. pongamiae (Syd. \& P. Syd.) Deighton on Pongamia sp. (Ellis 1976). Thus, the present specimen represents the third Asperisporium species on a host of the Fabaceae, and the first on a Galactia species.

Asperisporium cassiae shows longer multiseptate conidiophores and smaller conidia than the new species; while $A$. pongamiae has smaller conidia with smooth, wrinkled or minutely verruculose wall. Among other cercosporoid fungi on members of the genus Galactia, there are only two that, however, are typical Cercospora species, C. flagellifera G.F. Atk. and C. galactiae Ellis \& Everh. Thus, the well-defined and peculiar characteristics of the new species, such as verrucose, sublunate to fusoid conidia, infecting for the first time a Galactia species, differentiate this specimen from the remaining species of Asperisporium, clearly indicating that it belongs to a new species.

Pseudocercospora acosmii-subelegantis A. Hern.-Gut., Z.M. Chaves \& Dianese, sp. nov.

Fig. 2 MycoBank MB 814799

Holotype: BRAZIL. Distrito Federal: Planaltina, Águas Emendadas Ecological Station, on leaves of Acosmium subelegans (Mohlenbr.) Yakovlev (Fabaceael Faboideae), 6 Mar 1995, M. Sanchez (UB - Mycol Col. 7397).

Etymology: acosmii-subelegnatis, derived from the host species Acosmium subelegans.

Diagnosis: Differs from P. luzianensis, also known from the Cerrado, in having nonsynnematous conidiophores and conidia with thickened walls and septa (vs. conidia with thin septa and walls in P. luzianensis). 
Description: Lesions 0.5-3 mm diam., amphigenous, punctiform, circular or irregular, dark brown, without a delimiting margin. Colonies exclusively hypophyllous, brown. Mycelium internal. Stromata absent. Conidiophores in fascicles with up to ten conidiophores emerging through stomata, straight or slightly curved, sometimes showing percurrent proliferations, 41-93 $\mu \mathrm{m}$ long and 3-5 $\mu \mathrm{m}$ wide, 1-5 septate, light brown olivaceous, smooth. Conidiogenous cells integrated, polyblastic, sympodial, sometimes geniculate and showing percurrent extensions; conidiogenous loci flat slightly pigmented. Conidia simple, non catenate, obclavate or fusoid, sometimes curved, obconically truncate to truncate at the base, with unthickened, but lightly pigmented 1.5-3 $\mu \mathrm{m}$ wide hilum, obtuse to rounded at the apex, walls and septa thickened, 32-82 $\mu \mathrm{m}$ long, 7-10 $\mu \mathrm{m}$ broad at the widest part, 1-4 $\mu \mathrm{m}$ at the base, 1-2 $\mu \mathrm{m}$ near the apex, 0-9-septate, olivaceous-brown, paler near the apex, smooth.

Pseudocercospora aquae-emendadasensis A. Hern.-Gut., Z.M. Chaves \& Dianese, sp. nov. Fig. 3 MycoBank MB 814800

Holotype: BRAZIL. Distrito Federal: Planaltina, Águas Emendadas Ecological Station, on leaves of Chamaecrista orbiculata (Benth.) H.S. Irwin \& Barneby (Fabaceae), 21 Feb 1997, M. Sanchez (UB - Mycol. Col. 13421).

Etymology: Specific epithet referring to the site of type collection.

Diagnosis: Differs from $P$. chamaecristigena in having shorter mononematous conidiophores

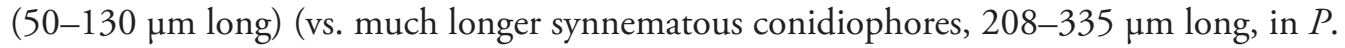
chamaecristigena).

Description: Lesions 1-4 mm diam., amphigenous, circular, brown to dark brown, surrounded by a yellowish halo. Colonies amphigenous, mainly caespitose, sometimes loosely synnematous, olivaceous-brown. Mycelium internal. Stromata 17-50 $\mu \mathrm{m}$ diam., moderately developed, textura globosa, substomatal, light brown. Conidiophores in loose to dense fascicles, emerging through stomata, slightly curved, somewhat flexuous or straight, 50-130 $\mu \mathrm{m}$ long, 5-7 $\mu \mathrm{m}$ broad at the widest part, 1-5-septate, occasionally with percurrent proliferation, light brown to olivaceous, smooth, thin-walled. Conidiogenous cells terminal, integrate, mostly monoblastic, sometimes polyblastic and sympodial, light olivaceous; conidiogenous loci aplanate, unthickened. Conidia solitary, straight, slightly curved, sometimes flexuous, obclavate to fusoid, obconically truncate, sometimes cylindrical at the base with slightly prominent hilum, $2-4 \mu \mathrm{m}$ wide, rounded to broadly rounded at the apex, 28-65 $\mu \mathrm{m}$ long, 5-7 $\mu \mathrm{m}$ broad at the widest part, 2-3 $\mu \mathrm{m}$ at the base, 2-3 $\mu \mathrm{m}$ near the apex, 0-4-septate, walls and septa thickened, sometimes constricted at some septa, light olivaceous to subhyaline, smooth. 
Additional specimen examined: BRAZIL. Distrito Federal: Planaltina, Águas Emmendadas Ecological Station, on leaves of Chamaecrista orbiculata (Fabaceae), 5 Mar 1997, M. Sanchez (UB - Mycol Col. 13703).

Pseudocercospora sennae A. Hern.-Gut., Z.M. Chaves \& Dianese, sp. nov.

Fig. 4 MycoBank MB 814801

Holotype: BRAZIL. Distrito Federal: Brasília, North Peninsula, on leaves of Senna rugosa (G. Don.) H.S. Irwin \& Barneby (Fabaceae), 31 May 1992, J.C. Dianese (UB - Mycol Col. 1132).

Etymology: Specific epithet derived from the host genus.

Diagnosis: Differs from P. taichungensis in having much longer, 1-5-septate conidiophores, 30-76 $\mu \mathrm{m}$ long, and much longer conidia, 24-132 $\mu \mathrm{m}$ (vs. conidiophores 10-25 × 1-3 $\mu \mathrm{m}, 0-2$-septate, conidia, 20-55 $\mathrm{m}$ long) in P. taichungensis).

Description: Lesions 1-6 mm diam., amphigenous, irregular, sometimes coalescent, brown with a darker margin and surrounded by a yellowish brown halo. Mycelium internal. Colonies amphigenous, brown. Stromata 42-183 $\mu \mathrm{m}$ diam., well-developed, textura angularis, erumpent, brown. Conidiophores in dense and compact layers arising from the stromata, unbranched, straight or slightly curved, cylindrical, 30-76 $\mu \mathrm{m}$ long, 4-6 $\mu \mathrm{m}$ broad at the widest part, reddish brown, 1-5-septate, sometimes percurrently proliferating, smooth, thin-walled. Conidiogenous cells terminal, integrated, mono- or polyblastic, sympodial; conidiogenous loci flat, unthickened, 3-3.5 $\mu \mathrm{m}$ wide. Conidia solitary, curved or straight, narrowly obclavate, sometimes cylindrical or filiform, obconically truncate to truncate at the base, hilum not thickened. 1-2.5 $\mu \mathrm{m}$ wide; obtuse to rounded or broadly rounded at the apex, 24-132 $\mu \mathrm{m}$ long, 3-5 $\mu \mathrm{m}$ broad at the widest part, $1-2 \mu \mathrm{m}$ at the base, $1-3 \mu \mathrm{m}$ near the apex, 1-8-septate, light brown to subhyaline, smooth, thin-walled.

Additional specimens examined: On leaves of Senna rugosa (Fabaceae) BRAZIL. Distrito Federal: Brasília, North Peninsula, 11 May 1992, J.C. Dianese (UB - Mycol Col. 1219). Planaltina, Águas Emendadas Ecological Station, 10 Jun 1992, R.B. Medeiros (UB - Mycol Col. 1139). Brazlândia, 18 Jun 1992, R.B. Medeiros (UB - Mycol Col. 1232). PADF, 18 Feb 1993, J.C. Dianese (UB - Mycol Col. 3177). Minas Gerais: Divinopolis, Expo Park, J.C. Dianese (UB - Mycol Col. 5514).

Pseudocercospora sennae-rugosae A. Hern.-Gut., Z.M. Chaves \& Dianese, sp. nov. Fig. 5 MycoBank MB 814802

Holotype: BRAZIL. Distrito Federal: Brasília, North Peninsula, on leaves of Senna rugosa (G. Don.) H.S. Irwin \& Barneby (Fabaceae), 9 Jan 1993, J.C. Dianese (UB - Mycol Col. 3033). 
Etymology: Epithet referring to the host species, Senna rugosa.

Diagnosis: Differs from $P$ sennae in having 2-12-septate conidiophores and longer, 3-18-septate conidia, 17-193 × 3-4 $\mu \mathrm{m}$ (vs. conidiophores 1-5-septate and conidia shorter, $24-132 \times 3-5 \mu \mathrm{m}$, with 1 to 8 septa in P. sennae).

Description: Lesions 2-8 mm diam., amphigenous, irregular, reddish brown, surrounded by a somewhat darker margin. Colonies mainly epiphyllous, occasionally hypophyllous, caespitose, shining grey. Stromata 152-245 $\mu \mathrm{m}$ diam., well-developed formed by cells of textura angularis at the lower part and textura prismatica at the upper part due to the compactly aggregated conidiophores, brown, subcuticular to subepidermal, erumpent. Conidiophores in very dense and compact fascicles to form a prismatic texture, individual conidiophores only evident at the apex or at conidiogenous cells, unbranched, straight or slightly curved, cylindrical, 50$66 \mu \mathrm{m}$ long, 4-5 $\mu \mathrm{m}$ broad at the widest part, 2-12-septate, brown, smooth, thin-walled. Conidiogenous cells terminal, integrated, monoblastic, narrowly lageniform to cylindrical, light brown; conidiogenous loci flat, unthickened, 2-2.5 $\mu \mathrm{m}$ wide. Conidia solitary, straight, slightly curved to strongly curved by being almost U-shaped, filiform to slightly obclavate; obconically truncate to truncate at the base, hilum unthickened, 1-2 $\mu \mathrm{m}$ wide, obtuse to rounded at the apex, 17-193 $\mu \mathrm{m}$ long, 3-4 $\mu \mathrm{m}$ broad at the widest part, 1-3 $\mu \mathrm{m}$ at the base and near the apex, 3-18-septate, light brown to light olivaceous-brown, smooth, thin-walled.

Additional specimen examined: On leaves of Senna rugosa (Fabaceae) BRAZIL. Distrito Federal: Planaltina, Núcleo Rural do Pipiripau, 10 Jun 1994, C.A. Inácio (UB - Mycol Col. 6191).

Pseudocercospora stryphnodendri A. Hern.-Gut., Z.M. Chaves \& Dianese, sp. nov. Fig. 6 MycoBank MB 814803

Holotype: BRAZIL. Minas Gerais: Divinópolis, Barrinha Farm, right side of Highway from Divinopólis to Formiga, 20¹3'54.9”S, 4505'33.7”W, on leaves of Stryphnodendron adstringens (Mart.) Coville (Fabaceae), 31 Dec 1991, J.C. Dianese (UB - Mycol Col. 894).

Etymology: Epithet referring to the host genus.

Diagnosis: Differs from the similar $P$. chamaecristae in having fasciculate, but nonsynnematous, much shorter conidiophores, 12-22 $\mu \mathrm{m}$ long and much longer and narrower, 3-9-septate conidia, 26-75 × 2-3 $\mu \mathrm{m}$ (vs. longer, synnematous conidiophores and much shorter and wider, 1-4-septate conidia, 25-35 × 5-8 $\mu \mathrm{m}$, in P. chamaecristae.

Description: Lesions 1-16 mm diam., amphigenous, irregular, sometimes circular, coalescent, purplish brown, without margin or surrounding halo. Colonies amphigenous, caespitose, yellowish grey. Stromata 13-26 $\mu \mathrm{m}$ diam., poorly developed, formed by cells of textura 
globosa, substomatal, light olivaceous to subhyaline. Conidiophores in compact stomatal fascicles, unbranched, straight or slightly curved, lageniform or ampulliform, 12-22 $\mu \mathrm{m}$ long, 3-4 $\mu \mathrm{m}$ broad at the widest part, $0-1$-septate, light olivaceous to subhyaline, smooth, thinwalled. Conidiogenous cells terminal, integrate, mainly forming a unicellular conidiophore, polyblastic, sympodial, geniculate, conidiogenous loci flat, unthickened, not pigmented, 1-1.5 $\mu \mathrm{m}$ wide. Conidia solitary, curved, flexuous or straight, filiform, obclavate, obconically truncate to truncate at the base, hilum unthickened, $1 \mu \mathrm{m}$ wide; acute, obtuse to rounded at the apex, $26-75 \times 2-3 \mu \mathrm{m}$ diam. at the widest part, $1-2 \mu \mathrm{m}$ at the base and near the apex, 3-9-septate, light olivaceous to subhyaline, smooth, thin-walled.

Additional specimens examined: on leaves of Stryphnodendron adstringens (Fabaceae), BRAZIL. Distrito Federal: Brasília, Vargem Bonita, Cerrado das Mansōes, 26 Jun 1994, M. Sanchez (UB - Mycol Col. 6222, 6223, 6242, 6243, and 6244). Planaltina, Águas Emendadas Ecological Station, 26 Jun 1995, Z.M. Chaves (UB - Mycol Col. 8880).

Pseudocercospora subcuticularis A. Hern.-Gut., Z.M. Chaves \& Dianese, sp. nov. Fig. 7 MycoBank MB 814804

Holotype: BRAZIL. Distrito Federal: Brasília, University of Brasília, Biological Experiment Station, on leaves of Senna rugosa (G. Don.) H.S. Irwin \& Barneby (Fabaceae), 11 Jul 1993, J.C. Dianese (UB - Mycol Col. 4092).

Etymology: Epithet referring to the subcuticular origin of the conidiophores.

Diagnosis: Differs from the similar P. sennae-rugosae in having 2-7-septate, shorter conidiophores (to $56 \mu \mathrm{m}$ long), and much shorter, 0-13-septate conidia (26-92 $\mu \mathrm{m}$ long) (vs. 2-12-septate conidiophores, 50-66 × 4-5 $\mu \mathrm{m}$, conidia 3-18-septate, 17-193 × 3-4 $\mu \mathrm{m}$ in P. sennae-rugosae).

Description: Lesions 3-18 mm diam., amphigenous, angular or irregular, limited by the leaf veins, brown, surrounded by a darker margin. Colonies amphigenous, dark greyish or black, mostly subcuticular. Stromata 125-225 $\mu \mathrm{m}$ diam., well-developed, intraepidermal, formed by cells of textura angularis, light brown or olivaceous. Conidiophores in dense fascicles, unbranched, straight or slightly curved, cylindrical, inclined towards the direction of the cuticle fissure, 33-56 $\mu \mathrm{m}$ long, 4-7 $\mu \mathrm{m}$ broad at the widest part, 2-7-septate, light olivaceous, smooth, thin-walled. Conidiogenous cells terminal, sometimes intercalary, integrated, monoblastic, cylindrical or slightly ampulliform, sometimes percurrently proliferating; condiogenous loci 2-3 $\mu \mathrm{m}$ wide. Conidia solitary, straight, sometimes curved, obclavate or fusoid to cylindrical, obconically truncate to truncate at the base, hilum unthickened, 1-4 $\mu \mathrm{m}$ wide; rounded to broadly rounded at the apex, 26-92 $\mu \mathrm{m}$ long, 4-7 $\mu \mathrm{m}$ broad at the widest part, $2-4 \mu \mathrm{m}$ at the base, $2-3 \mu \mathrm{m}$ near the apex, $0-13$-septate, light olivaceous, smooth, thin-walled. 
Comments to the new Pseudocercospora species: Six new Pseudocercospora species found on four host species belonging to three different genera, Acosmium, Chamaecrista, Stryphnodendron, and Senna, are described herein. The first species, $P$. acosmii-subelegantis, is characterized by the marked conidial polymorphism, all with thickened septa and walls, as present in some Pseudocercospora species previously allocated to Prathighada s. lat. The type species of the latter genus and other species with unthickened conidiogenous loci proved to belong to Pseudocercospora (Braun et al. 2013). Thus, the new species, which is clearly separated from Passalora acosmii A. Hern.-Gut \& Dianese, also found on $A$. subelegans (HernándezGutiérrez \& Dianese 2009), is here accommodated in Pseudocercospora s. str. There is no morphologically comparable species on allied hosts of the Amorpheae (dalbergioid clade, see Bruneau et al. 2013).

Pseudocercospora stryphnodendri is well-characterized by its short, pale, 0-1-septate conidiophores and narrow, very pale conidia. This is the first Pseudocercospora described on a host belonging to the genus Stryphnodendron. There are no comparable Pseudocercospora species on phylogenetically allied hosts of the Mimosoideae (Microlobium, Parapiptadenia, Pseudopiptadenia, Stryphnodendron lineage, see Jobson \& Luckow 2007).

The other new species occur on hosts of the genera Chamaechrista and Senna, both pertaining to a well-supported clade that is taxonomically usually referred to as Caesalpinioideae, tribe Cassieae, subtribe Cassiinae, comprising Cassia, Chamaecrista, and Senna, although the latter group probably warrants to be considered as tribe Cassieae s. str. (Bruneau et al. 2013). There are numerous Pseudocercospora spp. described on hosts of Cassia s. lat. (incl. Chamaecrista and Senna). A first comprehensive survey of and key to cercosporoid fungi on Cassia s. lat. was published by Braun (1989). He reduced Pseudocercospora cassia-occidentalis and $P$. singaporensis to synonymy with $P$. nigricans. An updated key to Pseudocercospora species, reflecting the morphological differences, is herein provided taking into account the summary shown in Table 1.

Table 1. Some morphotaxonomic features of the Pseudocercospora species found on hosts belonging to Cassia s. lat.

\begin{tabular}{|c|c|c|c|c|c|c|}
\hline \multirow[t]{2}{*}{ Pseudocercospora species } & \multirow{2}{*}{$\begin{array}{l}\text { Stromata } \\
\quad(\mu \mathrm{m})\end{array}$} & \multirow{2}{*}{$\begin{array}{l}\text { Conidiophores / } \\
\text { Fascicles }\end{array}$} & \multicolumn{2}{|c|}{ Conidiophores } & \multicolumn{2}{|c|}{ Conidia } \\
\hline & & & $(\mu \mathrm{m})$ & Septation & $(\mu \mathrm{m})$ & Septation \\
\hline P. angustata (2) & $S$ & $\begin{array}{l}\text { Mono, densely fas- } \\
\text { ciculate }\end{array}$ & $\begin{array}{l}10-50 \\
\times 2-3.5\end{array}$ & Rare & $\begin{array}{l}15-75 \\
\times 2-4\end{array}$ & $3-7$ \\
\hline P. aquae-emendadasensis (1) & $\begin{array}{l}17-50 \text {, text. globo- } \\
\text { sa, substomatal }\end{array}$ & Mono, stomatal & $\begin{array}{l}50-130 \\
\times 5-7\end{array}$ & $1-5$ & $\begin{array}{l}28-65 \\
\times 5-7\end{array}$ & $\begin{array}{l}0-4 \\
\text { Thick- } \\
\text { ened } \\
\text { wall } \\
\text { and } \\
\text { septa }\end{array}$ \\
\hline P. cassiae-alatae (3) & A & $\begin{array}{l}\text { Mono on external } \\
\text { hyphae }\end{array}$ & & $0-6$ & $15-90$ & $1-10$ \\
\hline P. cassiae-diphyllae (4) & $\begin{array}{l}\text { S, 3-5, substo- } \\
\text { matal }\end{array}$ & $\begin{array}{l}\text { Mono, stomatal and } \\
\text { laterally on creeping } \\
\text { hyphae }\end{array}$ & $\begin{array}{l}5-70 \\
\times 2.5-7\end{array}$ & $\begin{array}{l}\text { Asep- } \\
\text { tate }\end{array}$ & $\begin{array}{c}12-55 \\
\times 3-6\end{array}$ & $1-5$ \\
\hline
\end{tabular}


Table 1. (continued)

\begin{tabular}{|c|c|c|c|c|c|c|}
\hline \multirow[t]{2}{*}{ Pseudocercospora species } & \multirow{2}{*}{$\begin{array}{l}\text { Stromata } \\
\quad(\mu \mathrm{m})\end{array}$} & \multirow{2}{*}{$\begin{array}{l}\text { Conidiophores / } \\
\text { Fascicles }\end{array}$} & \multicolumn{2}{|c|}{ Conidiophores } & \multicolumn{2}{|c|}{ Conidia } \\
\hline & & & & Septation & $(\mu \mathrm{m}) \quad S e$ & Septation \\
\hline P. cassiae-occidentalis (5) & A & $\begin{array}{l}\text { Mono, fascicle with } \\
2-6 \text { conidiophores, } \\
\text { stomatal }\end{array}$ & $\begin{array}{l}60-130 \\
\times 4-5 \\
\mu \mathrm{m}\end{array}$ & $2-6$ & $\begin{array}{r}62-100 \\
\times 3.5-5\end{array}$ & $3-6$ \\
\hline P. cassiae-siameae (2) & $\begin{array}{l}\text { 17-24, substo- } \\
\text { matal }\end{array}$ & $\begin{array}{l}\text { Mono, fascicles com- } \\
\text { pact }\end{array}$ & $\begin{array}{l}15-27 \\
\times 3.5-4 \\
\text { non ge- } \\
\text { niculate }\end{array}$ & $0-1$ & $\begin{array}{l}29-94 \\
\times 3.5-4.5\end{array}$ & $5^{3-10}$ \\
\hline P. cassiae-sophorae (6) & $\begin{array}{l}\text { A or small on ex- } \\
\text { ternal hyphae }\end{array}$ & $\begin{array}{l}\text { Mono, conidio- } \\
\text { phores verruculose } \\
\text { single on external } \\
\text { hyphae, sometimes } \\
\text { subsynnematous }\end{array}$ & $\begin{array}{l}6.5-65 \\
\times 2-3.5\end{array}$ & $0-4$ & $\begin{array}{l}14-55 \times \\
2-3.5\end{array}$ & $2-5$ \\
\hline P. cassiigena (3) & $\begin{array}{l}\text { Globose } 25-40 \text {, } \\
\text { erumpent }\end{array}$ & $\begin{array}{l}\text { Mono, conidio- } \\
\text { phores densely fas- } \\
\text { ciculate }\end{array}$ & $\begin{array}{l}6-11 \\
\times 2-3\end{array}$ & 0 & $\begin{array}{l}22-36 \times \\
2-2.5\end{array}$ & $\begin{array}{l}\text { Strict- } \\
\text { ly } 3\end{array}$ \\
\hline P. cassia-fistulae (7) & $\begin{array}{l}\text { Elongate, erum- } \\
\text { pent, } \\
45 \text { wide }\end{array}$ & $\begin{array}{l}\text { Mono, fasciculate on } \\
\text { stromata, secondary } \\
\text { conidiophores sim- } \\
\text { ple on creeping hy- } \\
\text { phae originated from } \\
\text { stromata }\end{array}$ & $\begin{array}{l}10-30 \\
\times 2.5-5\end{array}$ & $0-2$ & $\begin{array}{l}25-65 \times \\
3-4\end{array}$ & $2-8$ \\
\hline P. caesalpiniicola (8) & $\begin{array}{l}30-45 \text {, substo- } \\
\text { matal }\end{array}$ & $\begin{array}{l}\text { Synnematous, fasci- } \\
\text { cle with up to } 35 \text { co- } \\
\text { nidiophores }\end{array}$ & $\begin{array}{l}50-251 \\
\times 4-6\end{array}$ & Multi & $\begin{array}{l}40-105 \times \\
5-6.5\end{array}$ & $\times \quad 2-9$ \\
\hline P. chamaecristae (8) & $\begin{array}{l}30-50, \text { substoma- } \\
\text { tal to intraepider- } \\
\text { mal }\end{array}$ & $\begin{array}{l}\text { Synnematous, } \\
\text { synnemata } 120-280 \\
\times 15-60\end{array}$ & & Multi & $\begin{array}{l}25-35 \times \\
5-8\end{array}$ & $1-4$ \\
\hline P. chamaecristigena (8) & $\begin{array}{l}\text { 35-83, substoma- } \\
\text { tal, textura globosa }\end{array}$ & $\begin{array}{l}\text { Synnematous, tex- } \\
\text { tura parallela, } \\
\text { synnemata } 208-335 \\
\times 20-83 \text {, with } 16- \\
65 \text { conidiophores }\end{array}$ & $\begin{array}{l}208-335 \\
\times 3-5\end{array}$ & $5 \quad 4-12$ & $\begin{array}{l}35-79 \times \\
5-8\end{array}$ & \\
\hline P. exilis (8) & $\begin{array}{l}25-50, \text { substo- } \\
\text { matal }\end{array}$ & $\begin{array}{l}\text { Synnematous, } \\
\text { synnemata 149-332 } \\
\times 7-23 \text {, with 5-13 } \\
\text { conidiophores }\end{array}$ & $\begin{array}{l}149-332 \\
\times 5-7\end{array}$ & $26-14$ & $\begin{array}{l}38-103 \times \\
6-9\end{array}$ & $\times \quad 4-10$ \\
\hline P. luzianensis (8) & $\begin{array}{l}\text { 50-88, substo- } \\
\text { matal }\end{array}$ & $\begin{array}{l}\text { Synnematous, } \\
\text { synnemata 315-600 } \\
\times 12-47 \text {, with 6-41 } \\
\text { conidiophores }\end{array}$ & $\begin{array}{l}141-600 \\
\times 3-5\end{array}$ & $\begin{array}{ll}0 & 8-21\end{array}$ & $\begin{array}{l}22-89 \times \\
5-7\end{array}$ & $1-8$ \\
\hline P. nigricans (2) & A & $\begin{array}{l}\text { Mono, stomatal, Fas- } \\
\text { cicle with } 2-12 \text { co- } \\
\text { nidiophores }\end{array}$ & $\begin{array}{r}15-125 \\
\times 3.5-5\end{array}$ & Multi & $\begin{array}{l}30-80 \times \\
3.5-5\end{array}$ & $3-5$ \\
\hline P. sennae (1) & $\begin{array}{l}\text { P, 42-183, text. } \\
\text { ang., erumpent }\end{array}$ & $\begin{array}{l}\text { Mono, fascicle com- } \\
\text { pact on stromata }\end{array}$ & $\begin{array}{l}30-76 \\
\times 4-6\end{array}$ & $1-5$ & $\begin{array}{l}24-132 \\
\times 3-5\end{array}$ & $1-8$ \\
\hline
\end{tabular}


Table 1. (continued)

\begin{tabular}{|c|c|c|c|c|c|c|}
\hline \multirow[t]{2}{*}{ Pseudocercospora species } & \multirow{2}{*}{$\begin{array}{l}\text { Stromata } \\
(\mu \mathrm{m})\end{array}$} & \multirow{2}{*}{$\begin{array}{l}\text { Conidiophores / } \\
\text { Fascicles }\end{array}$} & \multicolumn{2}{|c|}{ Conidiophores } & \multicolumn{2}{|c|}{ Conidia } \\
\hline & & & $(\mu \mathrm{m})$ & Septation & $(\mu \mathrm{m})$ & Septation \\
\hline P. sennae-rugosae (1) & $\begin{array}{l}152-245 / \text { textura } \\
\text { angularis at base, } \\
\text { prismatica above } \\
\text { subepidermal } \\
\text { erumpent }\end{array}$ & $\begin{array}{l}\text { Mono, fascicle com- } \\
\text { pact on stromata }\end{array}$ & $\begin{array}{l}50-66 \\
\times 4-5\end{array}$ & $2-12$ & $\begin{array}{l}17-193 \\
\times 3-4 \\
\text { Strong- } \\
\text { ly curved } \\
\text { almost u- } \\
\text { shaped }\end{array}$ & 3-18 \\
\hline P. sieberiana (9) & A & $\begin{array}{l}\text { Mono, fascicle with } \\
\text { up to } 12 \text { conidio- } \\
\text { phores, stomatal }\end{array}$ & $\begin{array}{l}37.5 \\
\times 2-2.5\end{array}$ & & $\begin{array}{l}27.5-72.5 \\
\times 2.5-3\end{array}$ & $51-6$ \\
\hline P. simulata (10) & A & $\begin{array}{l}\text { Mono, fascicle su- } \\
\text { perficial with } 2-20 \\
\text { conidiophores }\end{array}$ & $\begin{array}{l}50-300 \\
\times 3-5\end{array}$ & Multi & $\begin{array}{l}20-80 \\
\times 3-7\end{array}$ & $\begin{array}{l}1-5 \\
(3)\end{array}$ \\
\hline P. singaporensis (3) & A & $\begin{array}{l}\text { Mono, fascicles with } \\
2-10 \text { conidiophores, } \\
\text { stomatal }\end{array}$ & $\begin{array}{l}31-77 \\
\times 4.5- \\
5.5\end{array}$ & $1-4$ & $\begin{array}{l}30-67 \\
\times 3.5\end{array}$ & $\begin{array}{l}1-4 \\
(3)\end{array}$ \\
\hline P. subcuticularis (1) & $\begin{array}{l}125-225, \text { textu- } \\
\text { ra angularis, erum- } \\
\text { pent intraepider- } \\
\text { mal }\end{array}$ & $\begin{array}{l}\text { Mono, fascicles } \\
\text { densely compacted, } \\
\text { on stromata }\end{array}$ & $\begin{array}{l}33-56 \\
\times 4-7\end{array}$ & $2-7$ & $\begin{array}{l}26-92 \\
\times 4-7\end{array}$ & $0-13$ \\
\hline P. taichungensis (7) & $\begin{array}{l}30-100 \text { wide, } \\
\text { erumpent }\end{array}$ & $\begin{array}{l}\text { Mono, fascicles } \\
\text { densely compact, on } \\
\text { stromata }\end{array}$ & $\begin{array}{l}10-25 \\
\times 1-3\end{array}$ & $0-2$ & $\begin{array}{l}20-55 \\
\times 1.5-3\end{array}$ & $1-6$ \\
\hline
\end{tabular}

(1) Present study, (2) Deighton (1976), (3) Yen \& Lim (1980), (4) Braun (1989), (5) Yen (1981), (6) Singh et al. (2000), (7) Hsieh \& Goh (1990), (8) Hernández-Gutiérrez \& Dianese (2009), (9) Ram \& Mallaiah (1992), (10) Castañeda-Ruiz \& Braun (1989)

A - absent

$S$ - small, no dimensions offered

Mono - mononematous

Multi - multiseptate, no number offered

Key to Pseudocercospora species on hosts of the genus Cassia s. lat., including Chamaecrista and Senna species

1 Conidiophores synnematous $\ldots \ldots \ldots \ldots \ldots \ldots \ldots \ldots \ldots \ldots \ldots \ldots \ldots \ldots \ldots \ldots \ldots$

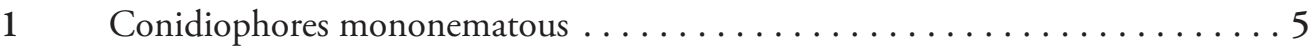

2(1) Synnemata 315-600 $\mu \mathrm{m}$ long; conidiophores 8-21-septate; conidia 1-8-septate,

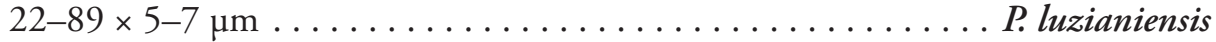

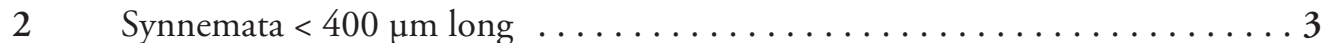

3(2) Synnemata 120-280 × 15-60; conidia 1-4-septate, $25-35 \times 5-8 \mu \mathrm{m}$; stromata 30-50 $\mu \mathrm{m}$ diam., substomatal to intraepidermal ......... P. chamaecristae

3 Synnemata $>300 \mu \mathrm{m}$ long; conidia $4-12$-septate $\ldots \ldots \ldots \ldots \ldots \ldots$ 
4(3) Synnemata composed of 5-13 conidiophores, 149-332 7 7-23 $\mu \mathrm{m}$; conidiophores 6-14-septate; conidia 4-10-septate, 38-103 × 6-9 $\mu \mathrm{m}$; stromata 25-50 $\mu \mathrm{m}$, substomatal, textura globosa ............... exilis

4 Synnemata composed of 16-65 conidiophores, 208-335 $\times 20-83 \mu \mathrm{m}$; conidia 4-12septate, 35-79 $\times 5-8 \mu \mathrm{m}$; stromata, 35-83 $\mu \mathrm{m}$ diam., substomatal, textura globosa P. chamaecristigena

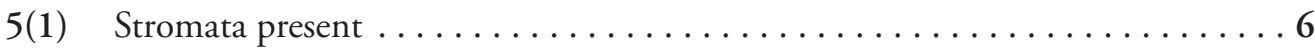

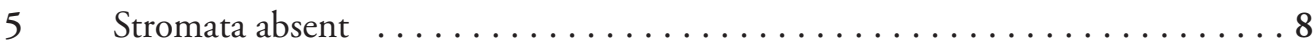

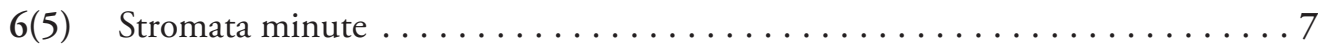

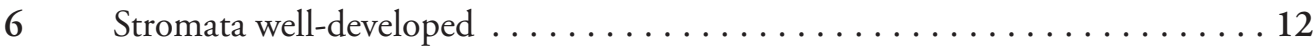

7(6) Stromatasmall; condiophores mostly on external hyphae, densely fasciculate; conidia 3-7-septate, up to $75 \mu \mathrm{m}$ long .............. angustata

7 Stromata3-5 $\mu \mathrm{m}$ diam.; conidiophores on stomatal fascicles and laterally on creeping hyphae, conidia 1-5-septate, up to $55 \mu \mathrm{m}$ long ......... P. cassiae-diphyllae

8(5) Conidiophores in fascicles, exclusively emerging through stomata . . . . . . . 9

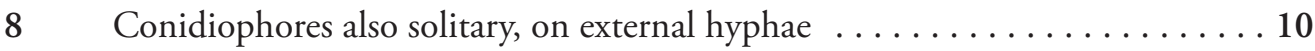

9(8) Conidiophores short, $37.5 \times 2-2.5 \mu \mathrm{m}$; conidia 1-6 septate, narrow, 27.5-72.5 $\times$ $2.5-3 \mu \mathrm{m} \ldots \ldots \ldots \ldots \ldots \ldots \ldots \ldots$. sieberiana

9 Conidiophores $15-130 \times 3.5-5.5 \mu \mathrm{m}$; conidia 1-6-septate, 30-100 × 3.5-5 $\mu \mathrm{m}$ P. nigricans (incl. P. cassiae-occidentalis, P. singaporensis)

10(8) Conidiophores up to $300 \mu \mathrm{m}$ long; conidia mostly 1-5-septate, 20-80 × 3-7 $\mu \mathrm{m}$

10 Conidiophores up to $80 \mu \mathrm{m}$ long $\ldots \ldots \ldots \ldots \ldots \ldots \ldots \ldots \ldots \ldots$

11(10) Conidia 1-10-septate, $15-90 \mu \mathrm{m}$ long . . . . . . . . . . . P. cassiae-alatae

11 Conidia 2-5-septate, $14-55 \mu \mathrm{m}$ long ............. cassiae-sophorae

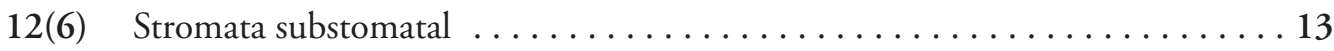

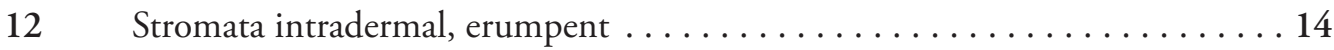

13(12) Stromata up to $50 \mu \mathrm{m}$ diam.; conidiophores 1 -5-septate, 50-130 $\times 5-7 \mu \mathrm{m}$, conidia thick walled, 0-4-septate, $28-65 \times 5-7 \mu \mathrm{m} \quad \ldots \ldots \ldots$ P. aquae-emendadasensis

13 Stromata up to $25 \mu \mathrm{m}$ diam.; conidiophores 0-1-septate, 15-27 $\times 3.5-4 \mu \mathrm{m}$, conidia 3-10-septate, $29-94 \times 3.5-4.5 \mu \mathrm{m} \ldots \ldots \ldots$. . . cassiae-siameae

14(12) Stromata globose, 25-40 $\mu \mathrm{m}$ diam.; condioiphores aseptate, 6-11 $\times 2-3 \mu \mathrm{m}$; conidia strictly 3-septate, $22-36 \times 2-2.5 \mu \mathrm{m} \ldots \ldots \ldots \ldots \ldots$. cassiigena

14 Stromata elongated, $45 \mu \mathrm{m}$ wide, or globose, reaching more than $100 \mu \mathrm{m}$ in diam.

15(14) Stromata elongated; primary conidiophores fasciculate on stromata, secondary conidiophores on superficial hyphae originating from stromata; conidiophores 0-2-septate, $10-30 \times 2.5-5 \mu \mathrm{m}$; conidia 2-8-septate, 25-65 $\times$ 3-4 $\mu \mathrm{m}$ 
15 Stromata globose reaching over $200 \mu \mathrm{m}$ in diam. .............. 16

16(15) Conidia up to 18 -septate; stromata over $200 \mu \mathrm{m}$ diam. . . . . . . . . . . 17

16 Conidia up to 8 -septate; stromata reaching up to $183 \mu \mathrm{m}$ diam. . . . . . . . 18

17(16) Conidiophores 2-12-septate, 50-66 × 4-5 $\mu \mathrm{m}$; conidia 3-18-septate, 17-193 × $3-4 \mu \mathrm{m} \ldots \ldots \ldots \ldots \ldots \ldots \ldots \ldots \ldots \ldots \ldots . \ldots \ldots$. sennae-rugosae

17 Conidiophores 2-7-septate, 33-56 × 4-7 $\mu \mathrm{m}$; conidia 0-13-septate, 26-92 × 4-7 $\mu \mathrm{m} \ldots \ldots \ldots \ldots \ldots \ldots \ldots \ldots \ldots \ldots \ldots \ldots \ldots \ldots \ldots$. subcuticularis

18(16) Conidiophores 1-5-septate, 30-76 $\times$ 4-6 $\mu \mathrm{m}$, sometimes showing percurrent proliferations; conidia 1-8-septate, $24-132 \times 3-5 \mu \mathrm{m} \ldots \ldots \ldots \ldots$. sennae

18 Conidiophores 0-2-septate, 10-25 × 1-3 $\mu \mathrm{m}$; conidia 1-6-septate, $20-55 \times 1.5-$

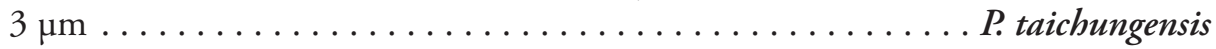

Sirosporium sclerolobii A. Hern.-Gut., Z.M. Chaves \& Dianese, sp. nov.

Fig. 8 MycoBank MB 814805

Holotype: BRAZIL. Goiás: Cristalina, Fazenda Nova Índia, on leaves of Sclerolobium paniculatum var. rubiginosum (Tul.) Benth. (Fabaceae), 10 Apr 1996, J.C. Dianese (UB Mycol Col. 4933).

Etymology: Specific epithet referring to the host genus, Sclerolobium.

Diagnosis: Differs from the similar S. munduleae in having 1-29-septate conidia, 15-107 $\mu \mathrm{m}$ long (vs. conidia up to 14 septate and up to $80 \mu \mathrm{m}$ long in $S$. munduleae).

Description: Lesions 2-13 mm diam., irregular, coalescent, amphigenous, dark brown, without a limiting margin. Colonies exclusively hypophyllous, caespitose, yellowish brown. Mycelium internal and external. Stromata 33-66 $\mu \mathrm{m}$ diam., well-developed, textura angularis, erumpent, light brown, originating from the internal mycelium. Primary conidiophores on top of the stromata, in loose fascicles, simple or branched, straight or slightly curved, narrowly lageniform to cylindrical, 16-33 $\mu \mathrm{m}$ long, 4-5 $\mu \mathrm{m}$ broad at the widest part, 0-5-septate, brown-olivaceous, smooth, thin-walled; conidiogenous cells terminal, integrated, monoor polyblastic, sympodial; conidiogenous loci conspicuous, thickened and dark. Secondary conidiophores formed as single polyblastic conidiogenous cells laterally differentiated on external hyphae, arising from stromata. Conidia solitary, curved, straight when young, later flexuous or sinuous, subcylindrical to obclavate, sometimes cylindrical, young conidia show thin walls, but mature conidia are thick-walled, often constricted at the septa; obconically truncate at the base, with a moderately thick, dark hilum, 1-2 um wide, rounded to broadly rounded at the apex, 15-107 $\mu \mathrm{m}$ long, 3-9 $\mu \mathrm{m}$ diam. at the widest part, 1-2 $\mu \mathrm{m}$ at the base, $2-4 \mu \mathrm{m}$ near the apex, 1-29-septate, occasionally with some oblique septa, olivebrown to light olive-brown, smooth. 
Additional specimen examined: on leaves of Sclerolobium paniculatum var. rubiginosum (Fabaceae). BRAZIL. Goiás: Cristalina, Fazenda Nova Índia, 26 Jun 1994, 10 Apr 1996, J.C. Dianese (UB - Mycol Col. 4934).

Comment: Braun et al. (2013) indicated that "the phylogenetic meaning and value of thick conidial walls and oblique to longitudinal septa as distinguishing characters between Sirosporium and Passalora are unclear", and further emphasized that the phylogenetic position of Sirosporium antenniforme (Berk. \& M.A. Curtis) Bubák \& Serebrian., the type species of genus, being still unknown. As the specimen on Sclerolobium paniculatum morphologically fits the traditional concept of the genus Sirosporium (Ellis 1971, 1976), and considering the currently unresolved phylogenetical position of this genus (Braun et al. 2013), it is advisable to place the new species in Sirosporium, at least tentatively. One of the most conspicuous characteristics of the specimen studied is the presence of fasciculate conidiophores on top of erumpent stromata and, at the same time, the presence of secondary conidiophores born laterally or apically on external hyphae, also originating from the stromata.

Amongst the 29 validly published Sirosporium names (http://indexfungorum.org, accessed on September 22, 2015), only three species were found on fabaceous hosts, viz. $S$. gliricidiae (Syd. \& P. Syd.) Deighton on Gliricidia sepium from the Philippines, S. munduleae (Syd. \& P. Syd.) M.B. Ellis on Mundulea suberosa from South Africa, and S. pluriseptatum (Gadp., C.D. Sharma, Firdousi, A.N. Rai \& K.M. Vyas) Kamal on Cassia fistula from India. Many of the known species associated with members of 16 other families show a similar conidial morphology, but all of them are easily separated from the new species based upon dimensional differences.

In Table 2 clear differences indicate that $S$. sclerolobii sp. nov., the fourth species found on Fabaceae, is easily distinguishable from the other three previously detected. Besides that, S. gliricidiae has dark stromata and the conidiophores on the adaxial leaf surface originate directly from the stromata, forming loose fascicles, but those of the abaxial surface, are solitary and originated directly from the superficial mycelium (Ellis 1976). Here in the case of $S$. sclerolobii the colonies are exclusively hypophyllous with both, conidiophore fascicles and external mycelium, formed on the same leaf surface.

Table 2. Comparison of Sirosporium species infecting Fabaceae, subfamily Faboideae

\begin{tabular}{lllll}
\hline Sirosporium species & \multicolumn{2}{c}{ Conidiophores } & \multicolumn{2}{c}{ Conidia } \\
& Size $(\mu \mathrm{m})$ & Septa & Size $(\mu \mathrm{m})$ & Septa \\
\hline S. gliricidiae & $-80 \times 4-8$ & $2-4$ & $40-85 \times 5-6$ & $5-13$ \\
S. munduleae & $-80 \times 5-8$ & $0-2$ & $22-45 \times 9-12$ & $1-6$ \\
S. pluriseptatum & $-101 \times 3-6.5$ & up to 14 & $37-208 \times 4-9$ & $2-35$ \\
S. sclerolobii, sp. nov. & $-66 \times 4-5$ & $0-5$ & $15-107 \times 3-9$ & $1-29$ \\
\hline
\end{tabular}


Sirosporium munduleae has longer (up to $80 \mu \mathrm{m}$ ) conidiophores with rugose to verrugose upper portion (Ellis 1976), different from the smooth, shorter ones (up to $66 \mu \mathrm{m}$ ) formed by $S$. sclerolobii. Finally, S. pluriseptatum has larger, up to 14 -septate conidiophores $(-101 \times$ 3-6.5 $\mu \mathrm{m})$ and much larger, 2-35-septate conidia $(37-208 \times 4-9 \mu \mathrm{m})$.

Zasmidium sclerolobii A. Hern.-Gut., Z.M. Chaves \& Dianese, sp. nov.

Fig. 9 MycoBank MB 814806

Holotype: BRAZIL. Goiás: Luziânia, Roberto Ronald Farm on road to Unaí, on leaves of Sclerolobium paniculatum Vogel (Fabaceae), 15 Nov 1994, J.C. Dianese (UB - Mycol. Col. 6838).

Etymology: Specific epithet referring to the host genus.

Diagnosis: Different from the similar $Z$. buteae by its very large stromata, shorter conidiophores, 30-74 $\mathrm{m}$ long, and shorter conidia 13-69 $\mu \mathrm{m}$ long, with 0-10 septa (vs. lacking stromata, much longer conidiophores, up to $150 \mu \mathrm{m}$, and much longer, 5-12 septate conidia, 64-157 $\mu \mathrm{m}$ long, in $Z$. buteae.

Description: Lesions 1-3 mm diam., amphigenous, circular or irregular, sometimes coalescent, dark brown, surrounded by a reddish margin. Colonies mainly hypophyllous, sometimes epiphyllous, caespitose, dark brown. Mycelium external and internal; external hyphae light brown, septate, verruculose to verrucose, branched, giving rise to holoblastic conidiogenous cells; internal hyphae subhyaline, septate, giving rise to stromata. Stromata well-developed, 44-125 um diam., textura globosa, subepidermal, erumpent, subhyaline to hyaline. Primary conidiophores on top of the stromata, in dense fascicles, simple, sometimes branched, straight or slightly curved, cylindrical, 30-74 $\mu \mathrm{m}$ long, 2-3 $\mu \mathrm{m}$ broad at the widest part, 2-8-septate, olive-brown, finely verruculose at the apex, thin-walled; secondary conidiophores differentiated as simples conidiogenous cells, arising laterally or terminally on creeping external verruculose hyphae. Conidiogenous cells terminal or intercalary, integrate, or conidiophores reduced to conidiogenous cells, mono- or polyblastic, sympodial, geniculate, verruculose, olivaceous; conidiogenous loci aplanate, thickened and dark, 1.5-2 $\mu \mathrm{m}$ wide. Conidia in simple or branched chains, straight to slightly curved, cylindrical, sometimes slightly obclavate, obconically truncate at the base with a thickened dark hilum, 1.5-2 $\mu \mathrm{m}$ wide, rounded to broadly rounded or conical-truncate at the apex, with 1-2 thick, dark apical scars in catenate conidia, 13-69 $\mu \mathrm{m}$ long, 3-5 $\mu \mathrm{m}$ broad at the widest part, 1-2 $\mu \mathrm{m}$ at the base, 1-3 $\mu \mathrm{m}$ near the apex, 0-10-septate, olivaceous, verruculose to verrucose, thin-walled.

Comment: Arzanlou et al. (2007) showed that although Zasmidium morphologically resembles Stenella, the type species of the latter genus clustered in the Teratosphaeriaceae, whereas Zasmidium in the Mycosphaerellaceae, as confirmed by Crous et al. (2009a, b) who described and molecularly characterized new Zasmidium species. Furthermore, conidia of Stenella (type species: S. araguata Syd.) have pileate conidiogenous loci (David 
1991), while those of Zasmidium [type-species: Z. cellare (Pers.) Fr.] and the Stenella-like species belonging in the Mycosphaerellaceae show thickened dark conidiogenous loci, a morphological character that correlates with the molecular data available (Arzanlou et al. 2007; Crous et al. 2009a, b; Braun et al. 2010).

In the following years a series of Stenella species with Cercospora-like conidiogenous loci, were recombined into Zasmidium, most of them based on morphological characteristics, reaching 198 species names in 2015 (http://www.indexfungorum.org/Names/Names.asp, Accessed on September 28, 2015), distributed in 68 different host families. The main host families with seven or more Zasmidium species are Fabaceae with 19 species, 13 in Myrtaceae and Rubiaceae, 10 in Rutaceae, 8 in Apocynaceae, 7 in Araceae and Asteraceae. The Zasmidium species on Fabaceae are distributed among 14 different host genera, six of them on Cassia species (Braun \& Urtiaga 2013).

There are no previous records of Zasmidium species on S. paniculatum. The following dichotomous key, based on Table 3, segregates those Zasmidium species that infect hosts belonging in family Fabaceae, most of them from India.

Table 3. Morphotaxonomic features of Zasmidium species, including the new Z. sclerolobii, found on host belonging in family Fabaceae

\begin{tabular}{|c|c|c|c|c|c|c|c|}
\hline \multirow{3}{*}{$\begin{array}{l}\text { Zasmidium species } \\
\text { Z. baubiniae (1) }\end{array}$} & \multirow{3}{*}{$\begin{array}{l}\text { Stromata } \\
\text { Present }\end{array}$} & \multirow{3}{*}{$\begin{array}{c}\begin{array}{c}\text { Conidiophores / } \\
\text { Fascicle* }\end{array} \\
\begin{array}{l}10-14 \text { condio- } \\
\text { phores/fascicle }\end{array}\end{array}$} & \multicolumn{2}{|c|}{ Conidiophores } & \multicolumn{3}{|c|}{ Conidia } \\
\hline & & & \multirow{2}{*}{$\begin{array}{l}(\mu \mathrm{m}) \mathrm{S} \\
16.5-69 \\
\times 2.5-3.5\end{array}$} & \multirow{2}{*}{$\begin{array}{l}\text { Septation } \\
0-5\end{array}$} & \multirow{2}{*}{$\begin{array}{l}(\mu \mathrm{m}) \quad \text { Sep } \\
\begin{array}{l}5-102.5 \\
\times 1.5-3\end{array}\end{array}$} & \multirow{2}{*}{$\begin{array}{r}\text { eptation } \\
0-10\end{array}$} & \multirow{2}{*}{$\begin{array}{c}\begin{array}{c}\text { Catenate / } \\
\text { Single }\end{array} \\
\text { S and C }\end{array}$} \\
\hline & & & & & & & \\
\hline Z. browneicola (2) & Lacking & $\mathrm{S}$ from $\mathrm{EH}^{*}$ & $\begin{array}{l}4.5-62.5 \\
\times 2.5-3\end{array}$ & $0-7$ & $\begin{array}{l}20-71 \\
\times 2.5-5.5\end{array}$ & $1-15$ & $\mathrm{C}$ \\
\hline Z. buteae (3) & $\begin{array}{l}13-16 \mu \mathrm{m} \\
\text { diam. }\end{array}$ & $S$ from $\mathrm{EH}$ & $\begin{array}{l}4-30 \\
\times 2-5\end{array}$ & $0-5$ & $\begin{array}{l}64-157 \\
\times 1-5\end{array}$ & $5-12$ & $\mathrm{C}$ \\
\hline Z. canavaliae (4) & Lacking & Fasciculate & $\begin{array}{r}30-300 \\
\times 3-4.5\end{array}$ & & $\begin{array}{l}10-50 \\
\times 3.0-5.5^{*}\end{array}$ & * $1-5$ & $\mathrm{C}$ \\
\hline Z. cassiae-fistulae (5) & $\begin{array}{l}\text { Substomatal } \\
10-30 \mu \mathrm{m} \\
\text { diam. }\end{array}$ & $\begin{array}{l}\text { Small loose fas- } \\
\text { cicles on super- } \\
\text { ficial stromata }\end{array}$ & $\begin{array}{r}40-150 \\
\times 2-4.5\end{array}$ & $\begin{array}{l}\mathrm{Plu}- \\
\text { risep- } \\
\text { tate }\end{array}$ & $\begin{array}{l}15-100 \\
\times 2-6\end{array}$ & $3-22$ & $S$ \\
\hline Z. cassiae-grandis (6) & Lacking & $S$ from $\mathrm{EH}$ & $\begin{array}{l}5-50 \\
\times 2.5-7\end{array}$ & $0-3$ & $\begin{array}{l}15-90 \\
\times 3-7\end{array}$ & $0-10$ & $\mathrm{~S}$ and $\mathrm{C}$ \\
\hline Z. cassiae-occidentalis (7) & $\begin{array}{l}\text { Present } 15- \\
20 \mu \mathrm{m} \text { diam. }\end{array}$ & $\begin{array}{l}\text { Fasciculate on } \\
\text { stromata or EH }\end{array}$ & $\begin{array}{r}48-140 \\
\times 2.5-5\end{array}$ & $2-6$ & $\begin{array}{l}20-80 \\
\times 2-7\end{array}$ & $1-4$ & $\mathrm{C}$ \\
\hline Z. cassiae-torae (8) & $\begin{array}{l}\text { Small 7-10 } \\
\mu \mathrm{m} \text { diam. }\end{array}$ & $\begin{array}{l}\text { Solitary or fas- } \\
\text { ciculate }\end{array}$ & $\begin{array}{l}3.5-54 \\
\times 3-5\end{array}$ & $1-5$ & $\begin{array}{l}17-76 \\
\times 3.5-5\end{array}$ & $1-5$ & $\mathrm{C}$ \\
\hline Z. cassiicola (9) & Lacking & $\mathrm{S}$ from $\mathrm{EH}$ & $\begin{array}{l}33-90 \\
\times 1.5-4\end{array}$ & $4-5$ & $\begin{array}{l}15-51 \\
\times 1.5-4.5\end{array}$ & $2-9$ & $\mathrm{C}$ \\
\hline Z. citri-griseum (10) & $\begin{array}{l}\text { Lacking or } \\
\text { small }\end{array}$ & $S$ from $E H$ & $\begin{array}{l}5-80 \\
\times 2.5-6\end{array}$ & $0-6$ & $\begin{array}{l}6-120 \\
\times 2-4.5\end{array}$ & $0-10$ & $\mathrm{~S}$ and $\mathrm{C}$ \\
\hline Z. crotalariicola $(11)$ & Lacking & $S$ from $\mathrm{EH}$ & $\begin{array}{l}80-140 \\
\times 4.0-5.0\end{array}$ & $2-4$ & $\begin{array}{l}22-75 \\
\times 3.5-5.0^{*}\end{array}$ & & $\mathrm{C}$ \\
\hline
\end{tabular}


Table 3. (continued)

\begin{tabular}{|c|c|c|c|c|c|c|c|}
\hline \multirow[t]{2}{*}{ Zasmidium species } & \multirow[t]{2}{*}{ Stromata } & \multirow{2}{*}{$\begin{array}{c}\text { Conidiophores / } \\
\text { Fascicle }^{*}\end{array}$} & \multicolumn{2}{|c|}{ Conidiophores } & \multicolumn{3}{|c|}{ Conidia } \\
\hline & & & $(\mu \mathrm{m})$ & Septation & $(\mu \mathrm{m}) \quad \mathrm{Se}$ & Septation & $\begin{array}{l}\text { Catenate / } \\
\text { Single }\end{array}$ \\
\hline Z. dalbergiae (12) & Lacking & $S$ from $\mathrm{EH}$ & $\begin{array}{l}45-290 \\
\times 3-4\end{array}$ & $3-11$ & $\begin{array}{l}48-105 \\
\times 2-3\end{array}$ & $1-3$ & $S$ and $C$ \\
\hline Z. fabaceaerum (13) & Lacking & $\mathrm{S}$ from $\mathrm{EH}$ & $\begin{array}{l}100-310 \\
\times 2-4\end{array}$ & & $\begin{array}{l}12-60 \\
\times 2-6\end{array}$ & $0-4$ & $\mathrm{C}$ \\
\hline Z. fabaceicola (14) & $\begin{array}{l}\text { Substomatal } \\
17-19 \mu \mathrm{m} \\
\text { diam. }\end{array}$ & $\begin{array}{l}\text { Fascicle from } \\
\text { stromata or ex- } \\
\text { ternal hyphae }\end{array}$ & $\begin{array}{r}75-210 \\
\times 1.5-5\end{array}$ & $4-12$ & $\begin{array}{l}13.5-40 \\
\times 1.5-5\end{array}$ & $0-3$ & $\mathrm{C}$ \\
\hline Z. millettiae (2) & Lacking & $S$ from $\mathrm{EH}$ & $\begin{array}{l}54-70 \\
\times 3.5-5.0\end{array}$ & $7-9$ & $\begin{array}{l}20-80 \\
\times 2.5-5.5\end{array}$ & $3-17$ & $7 \mathrm{C}$ \\
\hline Z. periandrae (15) & Lacking & $S$ from $\mathrm{EH}$ & $\begin{array}{l}10-80 \\
\times 3-5\end{array}$ & $\begin{array}{l}\text { Pluri- } \\
\text { sep- } \\
\text { tate }\end{array}$ & $\begin{array}{l}15-50 \\
\times 3-4\end{array}$ & $1-4$ & $\mathrm{C}$ \\
\hline Z. prosopidis (16) & $\begin{array}{l}10-75 \mu \mathrm{m} \\
\text { diam. }\end{array}$ & $\begin{array}{l}\text { Dense sporodo- } \\
\text { chial fascicles on } \\
\text { erumpent stro- } \\
\text { mata }\end{array}$ & $\begin{array}{l}5-40 \\
\times 2-5\end{array}$ & $0-1$ & $\begin{array}{l}20-110 \\
\times 2.5-5\end{array}$ & $0-14$ & $S$ \\
\hline Z. pterocarpigenum (17) & Lacking & Small fascicles & $\begin{array}{l}30-110 \\
\times 3-6\end{array}$ & & $\begin{array}{l}20-55 \\
\times 4-7\end{array}$ & $1-5$ & $\mathrm{C}$ \\
\hline Z. satpurense (18) & $2-8$ cells & $\begin{array}{l}\text { Fascicles with } \\
2-4 \text { conidio- } \\
\text { phores }\end{array}$ & $\begin{array}{l}25-72.5 \\
\times 3-4\end{array}$ & & $\begin{array}{l}10-42.5 \\
\times 2.5-5\end{array}$ & $1-8$ & $S$ \\
\hline Z. sclerolobii sp. nov. & $\begin{array}{l}44-125 \mu \mathrm{m} \\
\text { diam. }\end{array}$ & $\begin{array}{l}\text { Large stromatal } \\
\text { fascicles }\end{array}$ & $\begin{array}{l}30-74 \\
\times 2-3\end{array}$ & $2-8$ & $\begin{array}{l}13-69 \\
\times 3-5\end{array}$ & $0-10$ & $\mathrm{C}$ \\
\hline Z. tephrosiae (19) & Lacking & $\begin{array}{l}\text { Solitary or on } \\
\text { fascicle with } \\
2-15 \text { condio- } \\
\text { phores }\end{array}$ & $\begin{array}{l}15-150 \\
\times 3.5-5\end{array}$ & $\begin{array}{l}\text { Plu- } \\
\text { risep- } \\
\text { tate }\end{array}$ & $\begin{array}{l}40-130 \\
\times 3-5\end{array}$ & $\begin{array}{l}\text { Plu- } \\
\text { risep- } \\
\text { tate }\end{array}$ & $-S$ \\
\hline
\end{tabular}

(1) Haldar et al (2003), (2) Chaudhary et al. (2001), (3) Misra et al. (1997), (4) Deighton (1971), (5) Braun et al. (2003), (6) Braun \& Urtiaga (2013), (7) Kumar et al. (2006), (8) Singh et al. (2001), (9) Misra et al. (1999), (10) Fisher (1961), Braun et al. (2014), Huang et al. (2015), (11) Chaudhary et al. (1991), (12) Phengsintham et al. (2013), (13) Srivastava et al. (1994), (14) Kharwar et al. (2015), (15) Braun \& Freire (2006), (16) Heald \& Wolf (1911), and Braun et al. (2010), (17) Braun (2003), (18) Sharma et al. (2006), (19) Braun et al. (2010) $\mathrm{S}$ from $\mathrm{EH}=$ Solitary (mononematous) conidiophores originated from external hyphae

\section{Key to Zasmidium species on fabaceous hosts}

1 Stromata well-structured, reaching $125 \mu \mathrm{m}$ diam; conidiophores 2-8-septate, 30-74 $\times 2-3 \mu \mathrm{m}$, forming dense fascicle on top of the stromata; on Sclerolobium paniculatum,

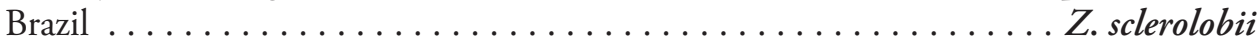

1 Stromata when present with a maximum of $75 \mu \mathrm{m}$ diam. . . . . . . . 2

2 Conidiophores fasciculate on top of stromata $\ldots \ldots \ldots \ldots \ldots \ldots$ 
2 Conidiophores with loose fascicles on poorly structured stromata, or single and mostly

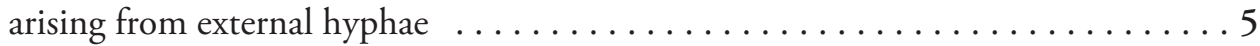

3 Conidiophores 2-6-septate, up to $140 \mu \mathrm{m}$ long; conidia 1-4-septate, 20-80 $\mu \mathrm{m}$ long; on Cassia occidentalis, Varanasi, India ............... cassiae-occidentalis

3 Conidiophores less than $70 \mu \mathrm{m}$ long; conidia up to 14 -septate, over $100 \mu \mathrm{m}$ long . . 4

4 Conidia catenate, 0-10-septate, 5-102.5 × 1.5-3 $\mathrm{m}$; on Bauhinia vahlii, Bengal,

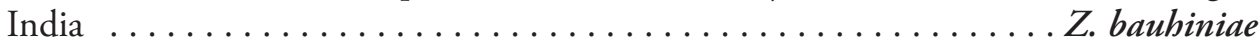

4 Conidia solitary, 0-14-septate, 20-110 $\times 2.5-5 \mu \mathrm{m}$; on Prosopis, USA . . Z. prosopidis

5 Conidiophores on poorly structured stromata $\ldots \ldots \ldots \ldots \ldots \ldots \ldots$

5 Condiophores solitary on external hyphae; stromata lacking $\ldots \ldots \ldots \ldots \ldots 8$

6 Conidiophores up to $150 \mu \mathrm{m}$ long; conidia solitary, 5-12 septate, 64-157 × 1-5 $\mu \mathrm{m}$; on Butea parviflora, Uttar Pradesh, India ................... Z. buteae

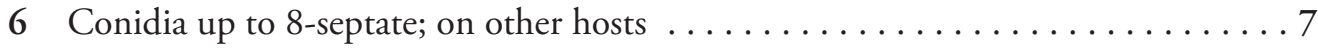

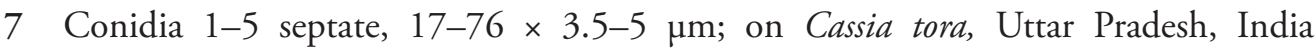
Z. cassiae-torae

7 Conidia 1-8-septate, $10-42.5 \times 2.5-5 \mu \mathrm{m}$; on Cassiia fistula, Madhia Pradesh, India Z. satpurense

8 Conidiophores over $300 \mu \mathrm{m}$ long; conidia 0-4-septate, 12-60 × 2-6 $\mu \mathrm{m}$; on Dolichos sp., Uttar Pradesh, India ..................... fabacearum

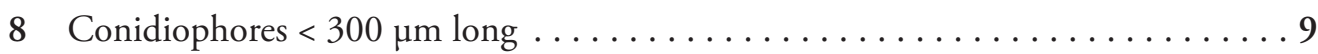

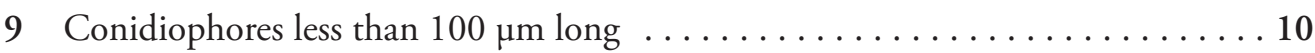

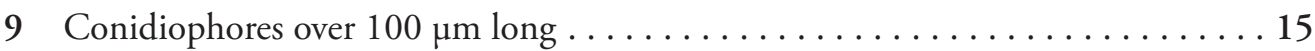

10 Conidia over $100 \mu \mathrm{m}$ long, 0-10-septate, $6-120 \times 2-4.5 \mu \mathrm{m}$; conidiophores 0-6-septate, 5-80 × 2.5-6 $\mu \mathrm{m}$; on Acacia mangium, Gabon, Africa Z. citri-griseum

10 Conidia less than $100 \mu \mathrm{m}$ long $\ldots \ldots \ldots \ldots \ldots \ldots \ldots \ldots \ldots \ldots \ldots \ldots \ldots \ldots \ldots$

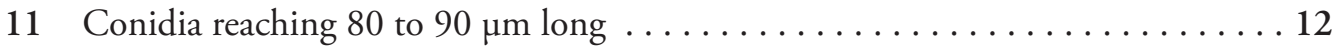

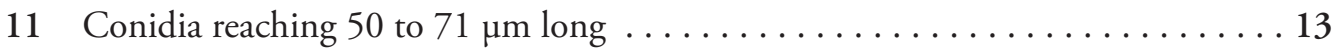

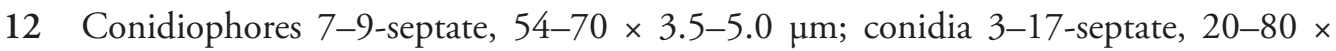

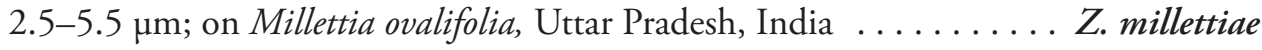

12 Conidiophores 0-3-septate, 5-50 × 2.5-7 $\mu \mathrm{m}$; conidia 0-10-septate, $15-90 \times 3-7$ $\mu \mathrm{m}$; on Cassia grandis, Cuba . . ................ cassiae-grandis

13 Conidiophores up to $90 \mu \mathrm{m}, 4-5$-septate, 33-90 × 1.5-4 $\mu \mathrm{m}$; conidia 2-9-septate,

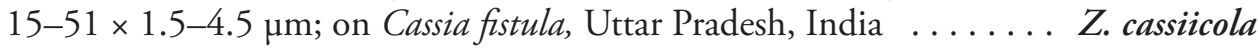

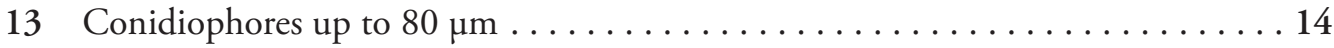

14 Conidiophores 4.5-62.5 × 2.5-3 $\mu \mathrm{m}$; conidia 1-15-septate, 20-71 × 2.5-5.5 $\mu \mathrm{m}$; on Brownea hybrida, Uttar Pradesh, India ................. Z. browneicola 
14 Conidiophores 10-80 × 3-5 $\mu \mathrm{m}$; conidia 1-4-septate, 15-50 ×3-4 $\mu \mathrm{m}$; on Periandra

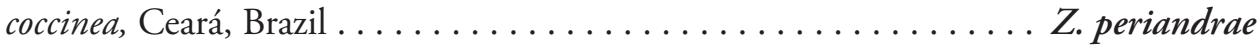

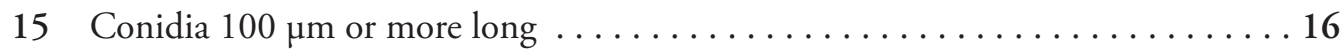

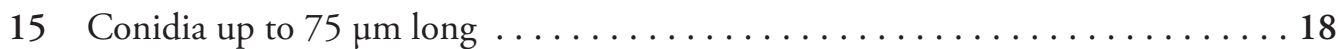

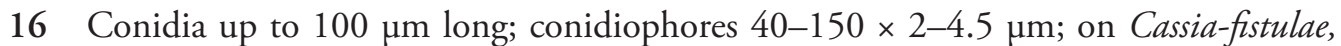
Uttar Pradesh, India . . . . . . . . . . . . . . cassia-fistulae

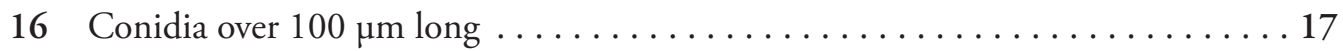

17 Conidia pluriseptate, 40-130 × 3-5 $\mu \mathrm{m}$; conidiophores $15-150 \times 3.5-5 \mu \mathrm{m}$; on Tephrosia hispidatae, Alabama, USA . . . . . . . . . . . . tephrosiae

17 Conidia, 48-105 × 2-3 $\mathrm{m}$; conidiophores 3-11-septate, 45-290 × 3-4 $\mu \mathrm{m}$; on Dalbergia cultrata, Laos ................... dalbergiae

18 Conidiophores over $200 \mu \mathrm{m}$ long . . . . . . . . . . . . . . . . . . . . 19

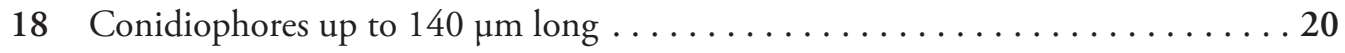

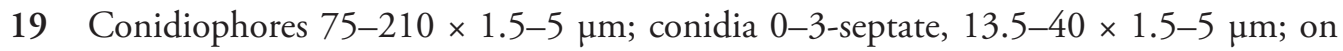
Vigna unguiculata, Chitwan, Nepal . . . . . . . . . . . Z. fabaceicola

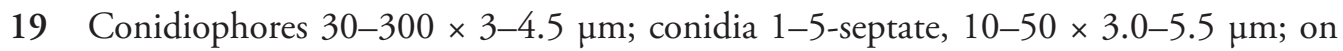
Canavalia ensiformis, Los Banos, Philippines . . . . . . . . . Z. canavaliae

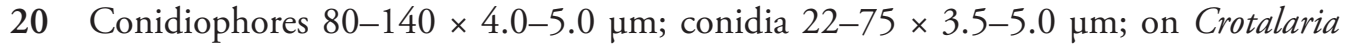
sp., Gorakhpur, India . . . . . . . . . . . . . . . . crotalariicola

20 Conidiophores 30-110 × 3-6 $\mu \mathrm{m}$; conidia 1-5-septate, 20-55 × 4-7 $\mu \mathrm{m}$; on Pterocarpus santalinus, Kerala, India . . . . . . . . . . Z. pterocarpigenum

Finally, following Braun et al. (2010), four cercosporoid Stenella species described on non-fabaceous hosts from the Brazilian Cerrado are herein transferred to Zasmidium because they are characterized by having typically cercosporoid conidiogenous loci and conidial hila, and rather cercosporoid conidia:

Zasmidium erythroxylicolum (Dorn.-Silva \& Dianese) Dorn.-Silva, A. Hern.-Gut. \& Dianese, comb. nov. (MycoBank, MB 814807)

Bas.: Stenella erythroxylicola Dorn.-Silva \& Dianese, Mycologia 99: 755, 2007.

Zasmidium erythroxyli-campestris (Dorn.-Silva, Pereira-Carv. \& Dianese) Dorn.-Silva, A. Hern.-Gut. \& Dianese, comb. nov. (MycoBank, MB 814808)

Bas.: Stenella erythroxyli-campestris Dorn.-Silva, Pereira-Carv. \& Dianese, Mycologia 99: $753,2007$.

Zasmidium erythroxyli-suberosi (Dorn.-Silva, Pereira-Carv. \& Dianese) Dorn.-Silva, A. Hern.-Gut. \& Dianese, comb. nov. (MycoBank, MB 814809)

Bas.: Stenella erythroxyli-suberosi Dorn.-Silva, Pereira-Carv. \& Dianese, Mycologia 99:755, 2007. 
Zamidium ocotei (Dorn.-Silva \& Dianese) Dorn.-Silva, A. Hern.-Gut. \& Dianese, comb. nov. (MycoBank, MB 814810)

Bas.: Stenella ocoteae Dorn.-Silva \& Dianese, Mycologia 99:759, 2007.

Acknowledgements. The authors thank CNPq for earlier support to the senior author and for fellowships via PPBIO/Cerrado-CNPq for the second and third authors. To Prof. Mariza Sanchez the authors show their appreciation for the work in the fungarium.

\section{References}

Arzanlou, M., Groenewald, J.Z., Gams, W., Braun, U., Shin, H-D. \& Crous, P.W. 2007. Phylogenetic and morphotaxonomic revision of Ramichloridium and allied genera. - Studies in Mycology 58: 57-93. http://dx.doi.org/10.3114/sim.2007.58.03

Barreto, R.W. \& Evans, H.C. 1995. The mycota of the weed Mikania micrantha in Southern Brazil with particular reference to fungal pathogens for biological control. - Mycological Research 99: 343-352. http://dx.doi:10.1016/S0953-7562(09)80911-8

Batista, A.C., Souza, R.G. \& Peres, G.E.P. 1960. Alguns Cercospora estudados no IMUR. - Publicaçóes do Instituto de Micologia da Universidade do Recife 266: 1-36.

Braun, U. 1989. Cercospora-like fungi on Cassia. - International Journal of Mycology and Lichenology 4: 191-204.

Braun, U. 2003. Miscellaneous notes on some cercosporoid hyphomycetes. - Bibliotheca Lichenologica 86: 79-98.

Braun, U. \& Freire, F.C.O. 2006. Some cercosporoid hyphomycetes from Brazil - IV. - Criptogamie, Mycologie 27: 231-248.

Braun, U. \& Urtiaga, R. 2013. New species and new records of cercosporoid hyphomycetes from Cuba and Venezuela (Part 3) - Mycosphere 4: 591-614. http://dx.doi.org/10.5943/mycosphere/4/2/3

Braun, U., Crous, P.W., Schubert, K. \& Shin, H-D. 2010. Some reallocations of Stenella species to Zasmidium. - Schlechtendalia 20: 99-104.

Braun, U., Nakashima, C. \& Crous, P.W. 2013. Cercosporoid fungi (Mycosphaerellaceae) 1. Species on other fungi, Pteridophyta and Gymnospermae. - IMA Fungus 4: 265-345. http://dx.doi.org/10.5598/ imafungus.2013.04.02.12

Braun, U., Crous, P.W. \& Nakashima, C. 2014. Cercosporoid fungi. (Mycosphaerellaceae) 2. Species on monocots (Acoraceae to Xyridaceae, excluding Poaceae). - IMA Fungus 5: 203-390. http://dx.doi. org/10.5598/imafungus.2014.05.02.04

Bruneau, A., Doyle, J.J., Herendeen, P. et al. 2013. Legume phylogeny and classification in the 21 st century: Progress, prospects and lessons for other species-rich clades. - Taxon 62: 217-248. http:// dx.doi.org/10.12705/622.8

Castañeda-Ruiz, R. \& Braun, U. 1989. Cercospora and allied genera of Cuba (I). - Cryptogamic Botany 1: 42-55.

Chaudhary, R., Chaturbhuji, G. \& Kamal. 1991. New species of Heteroconium, Psendocercospora and Stenella from India. - Mycological Research 95: 1070-1073. http://dx.doi.org/10.1016/S09537562(09)80548-0 
Chaudhary, R.K., Tripathi, M.S., Singh, P.N. \& Chaudhary, S. 2001. Novel taxa of Stenella from forest flora of Norh-Eastern Uttar Pradesh. - Indian Phytopahology 54: 226-232.

Chupp, J.C. 1954. A monograph of the fungus genus Cercospora. By the Author. Ithaca, New York.

Crous, P.W. \& Braun, U. 2003. Mycosphaerella and its anamorphs. 1. Names published in Cercospora and Passalora. CBS Biodiversity Series 1. Ponsen \& Looyen, Wageningen.

Crous, P.W., Alfenas, A.C. \& Barreto, R.W. 1997. Cercosporoid fungi from Brazil. 1. - Mycotaxon 64: 405-430.

Crous, P.W., Summerell, B.A., Carnegie, A.J., Wingfield, M.J. \& Groenewald, J.Z. 2009a. Novel species of Mycosphaerellaceae and Teratosphaeriaceae. - Persoonia 23: 119-146. http://dx.doi. org/10.3767/003158509X479531

Crous, P.W., Summerell, B.A., Carnegie, A.J., Wingfield, M.J., Hunter, G.C., Burgess, T.I., Andijc, V., Barber, P.A. \& Groenewald, J.Z. 2009b. Unravelling Mycosphaerella: do you believe in genera? Persoonia 23: 99-118. http://dx.doi.org/10.3767/003158509X479487

Crous, P.W., Schumacher, R.K., Wingfield, M.J., Lombardi, L., Giraldo, A., Christensen, M., Gardiennet, A., Nakashima, C., Pereira, O.L., Smith, A.J. \& Groenewald, J.Z. 2015. Fungal systematics and evolution: Fuse 1. - Sydowia 67: 81-118. http://dx.doi.org/10.3767/003158509X479487

David, J.C. 1991. Parastenella, a new generic name for Heterosporium magnolia. - Mycological Research 95: 123-128. http://dx.doi.org/10.1016/S0953-7562(09)81369-5

Deighton, F.C. 1971. Brown leaf mould of Canavalia caused by Stenella canavaliae (H. \& P. Syd.) comb. nov. - Transactions of the British Mycological Society 56: 411-418. http://dx.doi.org/10.1016/ S0007-1536(71)80133-X

Deighton, F.C. 1976. Studies on Cercospora and allied genera, VI. Pseudocercospora Speg., Pantospora Cif. and Cercoseptoria Petr. - Mycological Papers 140: 1-168. http://dx.doi.org/10.1016/S00071536(71)80133-X

Dianese, A.C., Costa, A.M. \& Dianese, J.C. 2008. A new Pseudocercospora species on Passiflora setácea. Mycotaxon 105: 1-5.

Dianese, J.C. \& Câmara, M.P.S. 1994. Pseudocercospora aspidospermatis, a new combination for Bactrodesmiella aspidospermatis. - Sydowia 46: 225-232.

Dornelo-Silva, D. \& Dianese, J.C. 2003. Hyphomycetes on Vochysiaceae from the Brazilian Cerrado. Mycologia 95: 1239-1251. http://dx.doi.org/10.2307/3761924

Dornelo-Silva, D., Pereira-Carvalho, R.C. \& Dianese, J.C. 2007. New Stenella and Parastenella species from the Brazilian Cerrado. - Mycologia 99: 753-764. http://dx.doi.org/10.3852/mycologia.99.5.753

Ellis, M.B. 1971. Dematiaceous Hyphomycetes. Commonwealth Mycological Institute, Kew, Surrey.

Ellis, M.B. 1976. More Dematiaceous Hyphomycetes. Commonwealth Mycological Institute, Kew, Surrey.

Fisher, F.E. 1961. Greasy spot and tar spot of citrus in Florida. - Phytopathology 51: 297-303.

Furlanetto, C. \& Dianese, J.C. 1999. Some Pseudocercospora species and a new Prathigada species from the Brazilian cerrado. - Mycological Research 103: 1203-1209. http://dx.doi.org/10.1017/ S0953756299008394

Haldar, D., Ray, J.B. \& Das, A.K. 2003. Two new Stenella species from India. - Journal of Mycopathological Research 41: 63-66.

Heald, F.D. \& Wolf, F.A. 1911. New species of Texas fungi. - Mycologia 3: 5-22. http://dx.doi. org/10.2307/3753651 
Hernández-Gutiérrez, A. \& Dianese, J.C. 2008. New cercosporoid fungi from the Brazilian Cerrado. 1. Species on hosts of the families Anacardiaceae, Araliaceae, Bombacaceae, Burseraceae and Celastraceae.Mycotaxon 106: 41-63.

Hernández-Gutiérrez, A. \& Dianese, J.C. 2009. New cercosporoid fungi from the Brazilian Cerrado 2. Species on hosts of the subfamilies Caesalpinoideae, Faboideae, and Mimosoideae (Leguminosae s. lat.). - Mycotaxon 107: 1-24. http://dx.doi.org/10.5248/107.1

Hernández-Gutiérrez, A. \& Dianese, J.C. 2014a. Cercosporoid hyphomycetes on malpighiaceous hosts from the Brazilian Cerrado: New Passalora and Pseudocercospora species on hosts of the genus Banisteriopsis. Mycological Progress 13: 365-371. http://dx.doi.org/10.1007/s11557-013-0922-6

Hernández-Gutiérrez, A. \& Dianese, J.C. 2014b. New Passalora species on Peixotoa (Malpighiaceae) from the Brazilian Cerrado. - Mycological Progress 13: 75-79. http://dx.doi.org/10.1007/s11557-013-0894-6 Hernández-Gutiérrez, A., Braun, U. \& Dianese, J.C. 2014. Cercosporoid hyphomycetes on malpighiaceous hosts from the Brazilian Cerrado: Species of Pseudocercospora on hosts belonging to Byrsonima. Mycological Progress 13: 193-210. http://dx.doi.org/10.1007/s11557-014-0971-5

Hsieh, W-H. \& Goh, T.-K. 1990. Cercospora and similar fungi from Taiwan. Maw Chang Book Co., Taipei. Huang, F., Groenewald, J.Z., Zhu, L., Crous, P.W. \& Li, H. 2015. Cercosporoid diseases of Citrus. Mycologia: preliminary version published on October 2, 2015, as doi:10.3852/15-059.

Inácio, C.A. \& Dianese, J.C. 1998. Foliicolous fungi on Tabebuia species. - Mycological Research 102: 695-708. http://dx.doi.org/10.1017/S0953756297005856

Inácio, C.A. \& Dianese, J.C. 1999. A new Mycovellosiella species on Myracrodruon urundeuva. - Mycotaxon 72: 251-254.

Inácio, C.A. \& Dianese, J.C. 2006. Foliicolous fungi on Tabebuia species from the cerrado. - Mycological Progress 5: 120-127. http://dx.doi.org/10.1007/s11557-006-0507-8

Inácio, C.A., Furlanetto, C., Hernández-Gutiérrez, A. \& Dianese, J.C. 1996. Some Cercospora species originally described by Ahmés Pinto Viégas. - Fitopatologia Brasileira 21: 405-409.

Jobson, R.W. \& Luclow, M. 2007. Phylogenetic studies of the genus Piptadenia (Mimosoideae: Leguminosae) using plastid trnL-F and trnK/matK sequence data. - Systematic Botany 32: 569-575. http://dx.doi. org/10.1600/036364407782250544

Kharwar, R.N., Singh, A., Singh, R. \& Kumar, S. 2015. Two new species of Zasmidium from Nepal. Mycotaxon 130: 241-246. http://dx.doi.org/10.5248/130.241

Kumar, A., Kumar, A. \& Kharwar, R.N. 2006. Two new phytoparasitic hyphomycetes from Varanasi, India. - Indian Phytopathology 59: 85-90.

Medeiros, R.B. \& Dianese, J.C. 1994. Passalora eitenii sp. nov. on Syagrus comosa (Mart.) Mart. in Central Brazil, and a key for identification of Passalora species. - Mycotaxon 51: 509-513.

Mendonça, R.C., Felfili, J.M., Walter, B.M.T., Silva-Júnior, M.C., Rezende, A.V., Filgueiras, T.S. \& Nogueira, P.E. 2008. Flora vascular do Cerrado. - In: S.M. Sano \& S.P. Almeida (eds). Cerrado: ambiente e flora. Pp. 289-556. EMBRAPA-CPAC, Planaltina.

Minnis, A.M., Kennedy, A.H., Grenier, D.B., Rehner, S.A. \& Bischoff, J.F. 2011. Asperisporium and Pantospora (Mycosphaerellaceae): epitypifications and phylogenetic placement. - Persoonia 27: 1-8. http://dx.doi.org/10.3767/003158511X602071

Misra, S., Srivastava, N. \& Srivastava, A.K. 1997. New species of Stenella from India. - Mycological Research 101: 278-280. http://dx.doi.org/10.1017/S0953756296002572 
Misra, S., Srivastava, A.K. \& Kamal. 1999. Further additions to Stenella from India and Nepal. - Mycological Research 103: 268-270. http://dx.doi.org/10.1017/S0953756298006108

Phengsintham, P., Chukeatirote, E., McKenzie, E.H.C., Hyde, K.D. \& Braun, U. 2013. Monograph of Cercosporoid fungi from Laos. - Current Research in Environmental \& Applied Mycology 3: 34-158. http://dx.doi.org/10.5943/cream/3/1/2.

Ram, M.R. \& Mallaiah, K.V. 1992. Pseudocercospora sieberiana sp. nov. from India. - Mycotaxon 45: 405-408.

Sharma, N., Soni, K.K. \& Verma, R.K. 2006. Some new hyphomycetes from forests of Satpura. - Indian Journal of Tropical Biodiversity 14: 34-40.

Singh, S.Y.K. \& Bhalla, K. 2000. New Pseudocercospora species causing foliar diseases in plain forests of Vindhya region in India. - Indian Phytopathology 53: 399-403.

Singh, S.Y.K. \& Bhalla, K. 2001. Four new foiiicolous hyphomycetes from Vindhya Hills, India. - Journal of Mycology and Plant Pathology 31: 277-286.

Srivastava, K., Srivastava, A.K. \& Kamal. 1994. New species of Stenella from India. - Mycological Research 98: 516-520. http://dx.doi.org/10.1016/S0953-7562(09)80470-X

Viégas, A.P. 1945. Alguns fungos do Brasil: Cercosporae. - Boletim Sociedade Brasileira de Agronomia 8: $1-160$.

Yen, J.M. 1981. Étude sur les champignons parasites de Sud-Est asiatique, 41. Les Cercospora de Formosae.Bulletin de la Société Mycologique de France 97: 91-96.

Yen, J.M. \& Lim, G. 1980. Cercospora and allied genera of Singapore and the Malay Peninsula. - The Gardens' Bulletin Singapore 33: 151-263. 


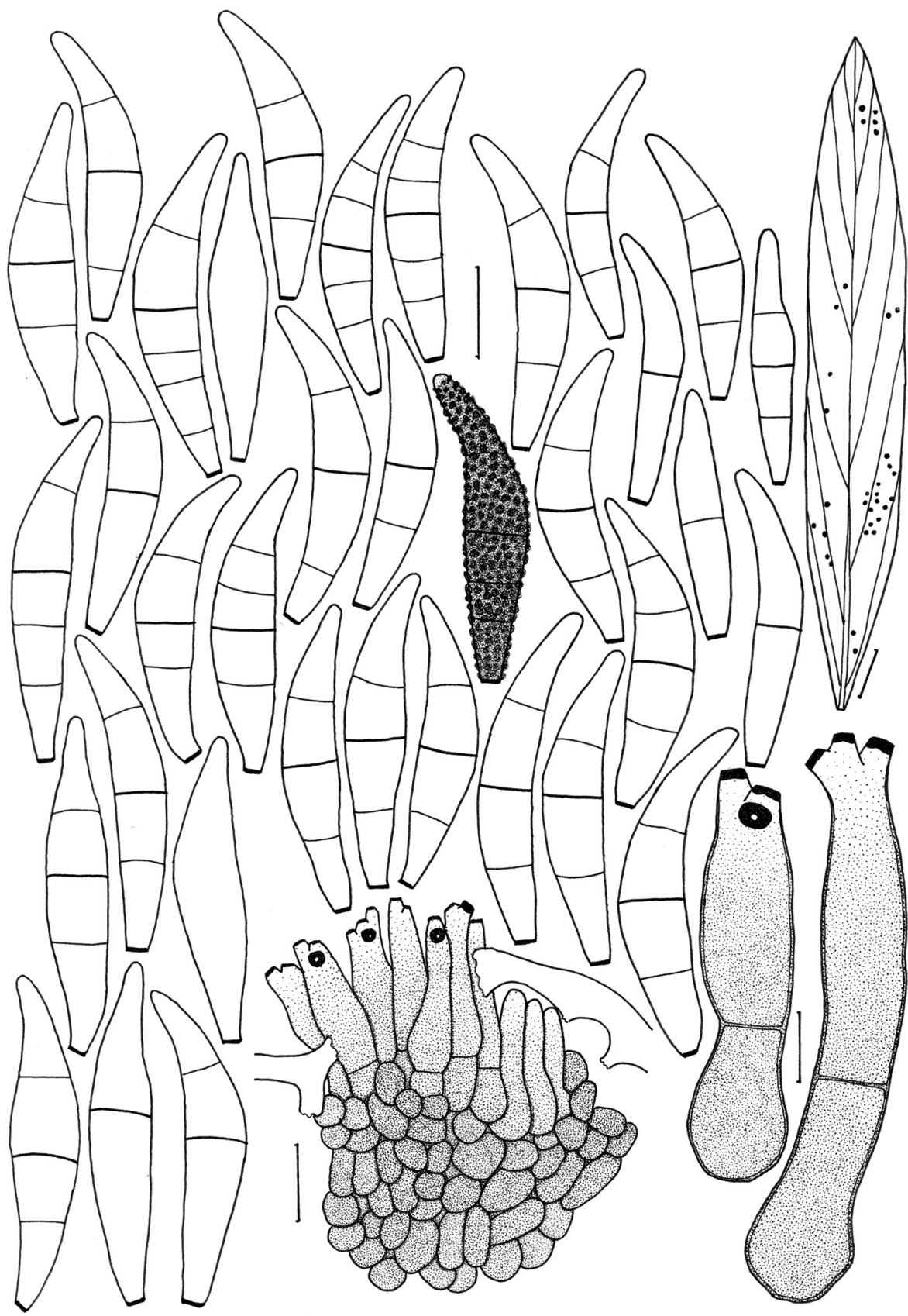

Fig. 1. Asperisporium chapadensis on Galactia peduncularis. Lesions on a leaf (top right) $($ scale $=10 \mathrm{~mm})$; erumpent-subcuticular stroma with a fascicle of primary conidiophores $($ scale $=10 \mu \mathrm{m})$; detailed view of a conidiophore bearing conidiogenous cells with protuberant thickened loci (right) (scale $5 \mu \mathrm{m}$ ); representative sample of the conidia. (scale $=10$ $\mu \mathrm{m})$. Based on the holotype UB - Mycol. Col. 11332. 


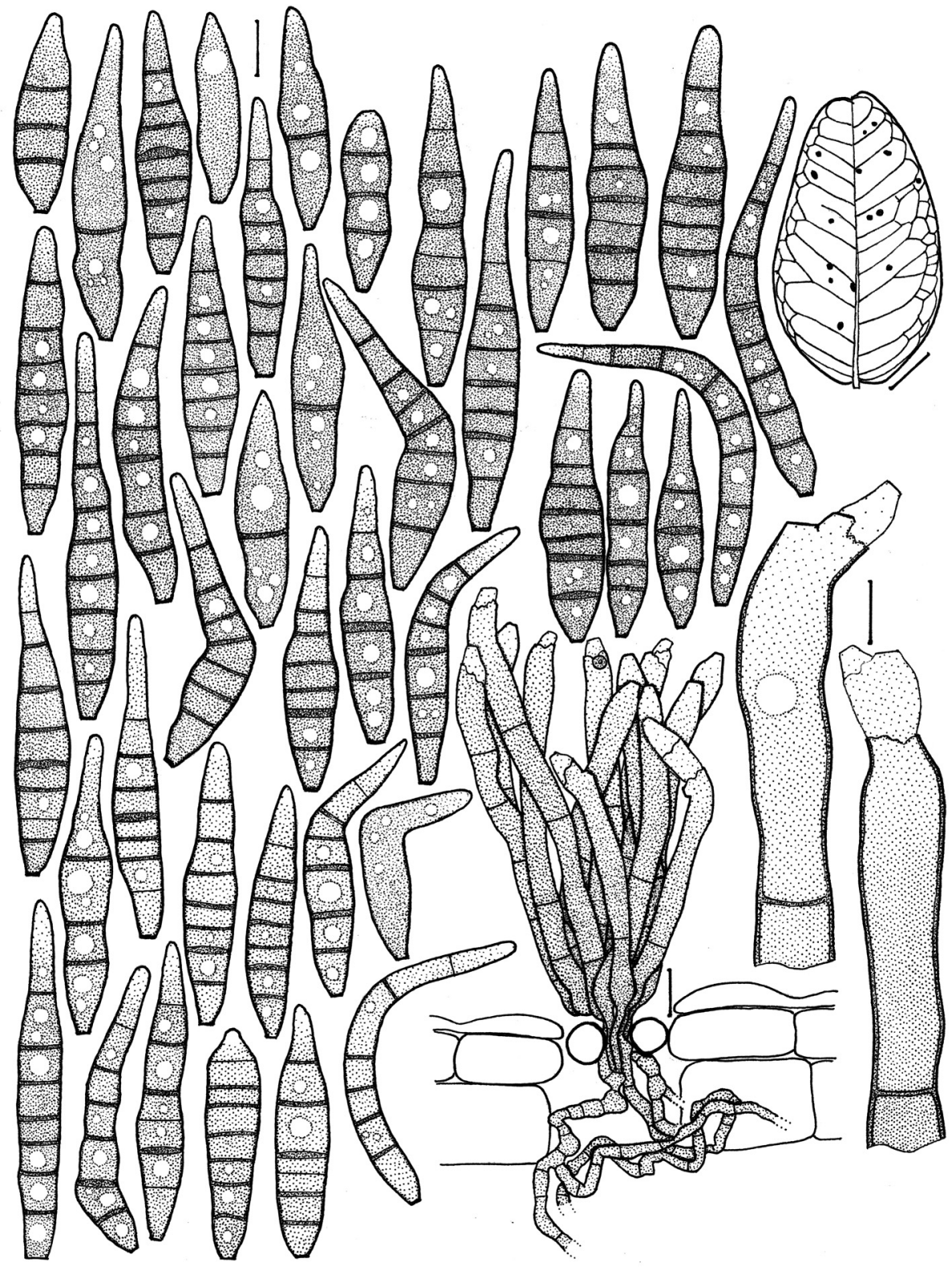

Fig. 2. Pseudocercospora acosmii-subelegantis on Acosmium subelegans. Lesions on a leaf (top right) $($ scale $=10 \mathrm{~mm})$; stomatal condiophore fascicle originated from internal hyphae $($ scale $=10 \mu \mathrm{m})$; a representative sample of conidia with thickened walls and septa (scale $=$ $10 \mu \mathrm{m}$ ); at the right side of the conidiophore fascicle, a detailed view of two conidiogenous cells showing aplanate conidiogenous loci $($ scale $=5 \mu \mathrm{m})$. Based on the holotype UB $-\mathrm{My}-$ col. Col. 7397. 


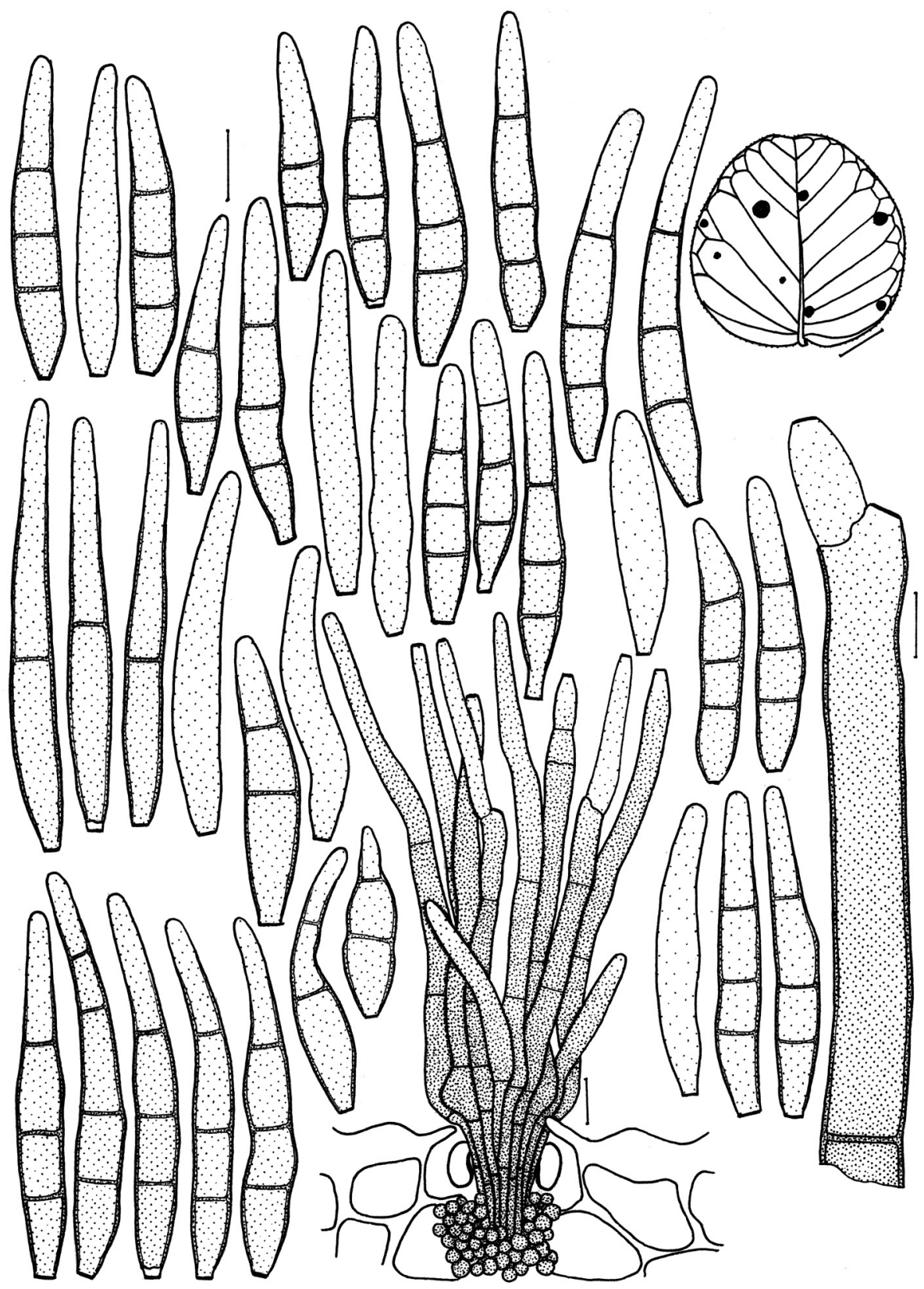

Fig. 3. Pseudocercospora aquae-emendadasensis on Chamaecrista orbiculata. Lesions on a leaf (top right) $($ scale $=10 \mathrm{~mm}$ ); stomatal condiophore fascicle originated from internal hyphae $($ scale $=10 \mu \mathrm{m})$; a representative sample of conidia with thickened walls and septa (scale $=$ $10 \mu \mathrm{m}$ ); at the right side of the conidiophore fascicle, a detailed view of a conidiogenous cell showing aplanate conidiogenous loci $($ scale $=5 \mu \mathrm{m})$. Based on the holotype UB $-\mathrm{My}-$ col. Col. 13421. 


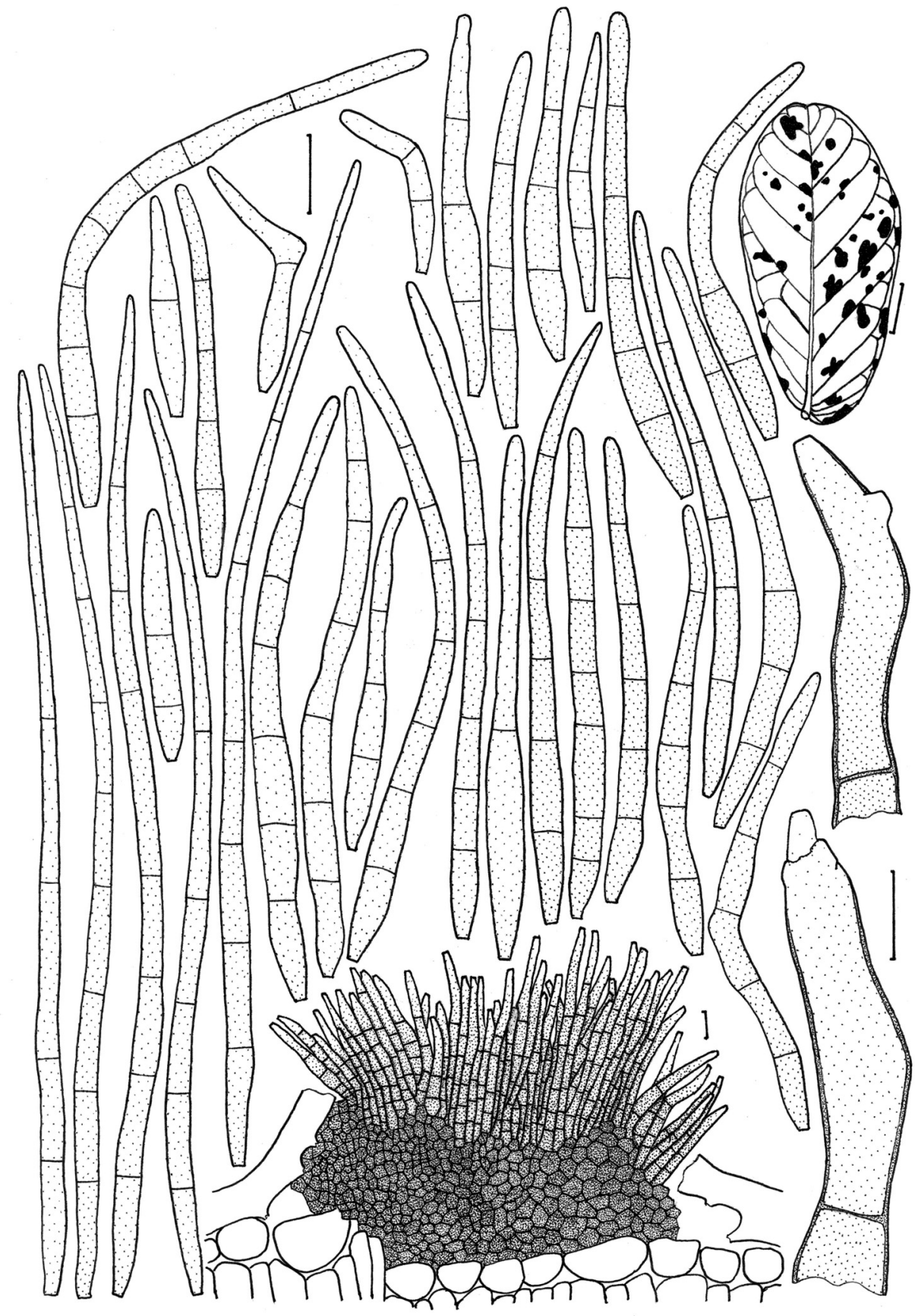

Fig. 4. Pseudocercospora sennae on Senna rugosa. Lesions on a leaf (top right) (scale $=10$ $\mathrm{mm}$ ); erumpent-subepidermal wide stroma bearing thightly packed conidiophores (scale $=$ $10 \mu \mathrm{m})$; a representative sample of polymorphic conidia $($ scale $=10 \mu \mathrm{m})$; at the bottm right, detailed view of a conidiogenous cell (scale $=5 \mu \mathrm{m})$. Based on the holotype UB - Mycol. Col. 1132. 


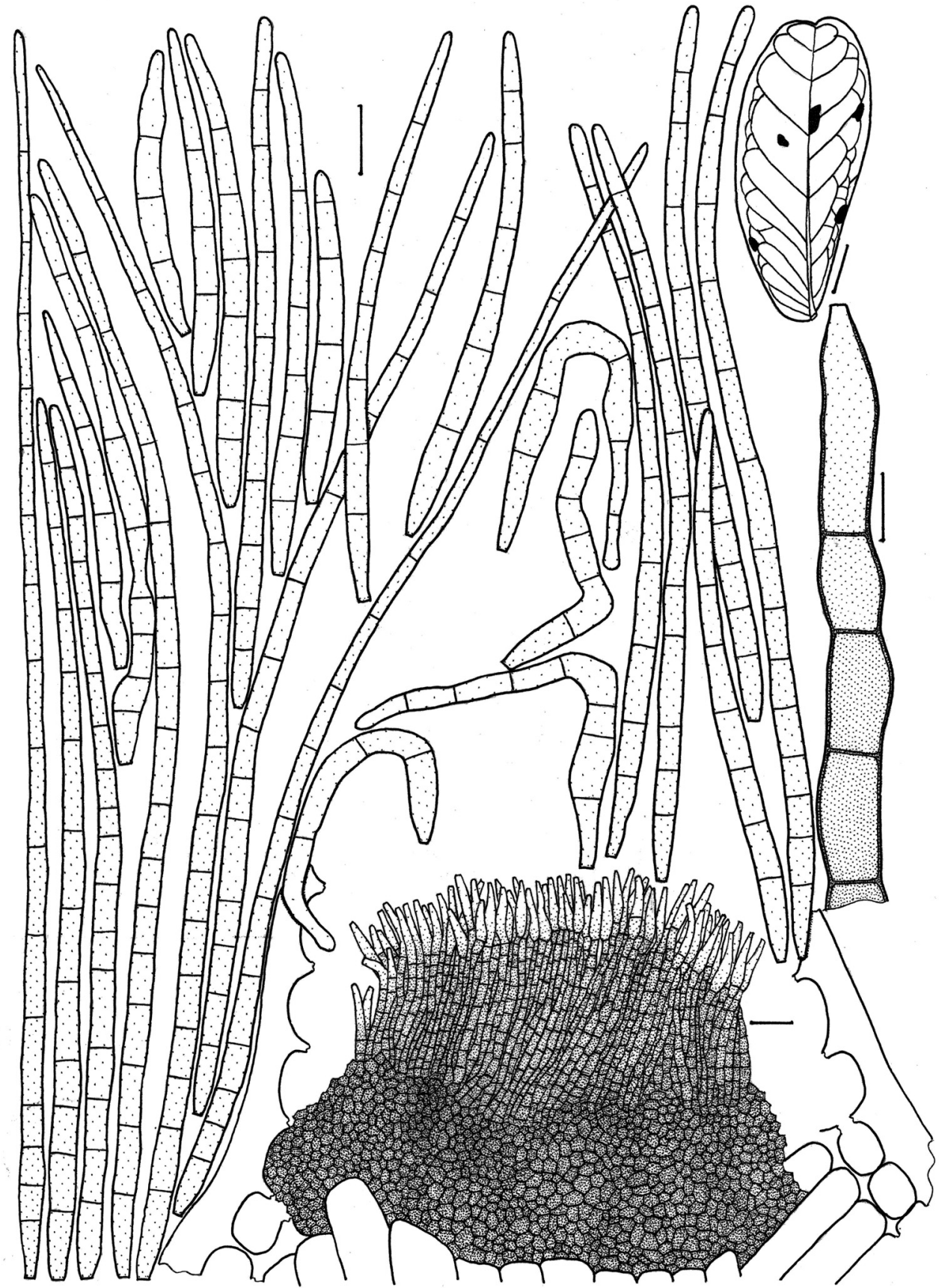

Fig. 5. Pseudocercospora sennae-rugosae on Senna rugosa. Lesions on a leaf (top right) (scale $=10 \mathrm{~mm}$ ); erumpent subepidermal stroma bearing a compact fascicle of highly septate conidiophores $($ scale $=10 \mu \mathrm{m})$; a representative sample of conidia $($ scale $=10 \mu \mathrm{m})$; a pluriseptate conidiophore with an apical conidiogenous cell (bottom right) (scale $=5 \mu \mathrm{m})$. Based on the holotype UB - Mycol. Col. 3033. 


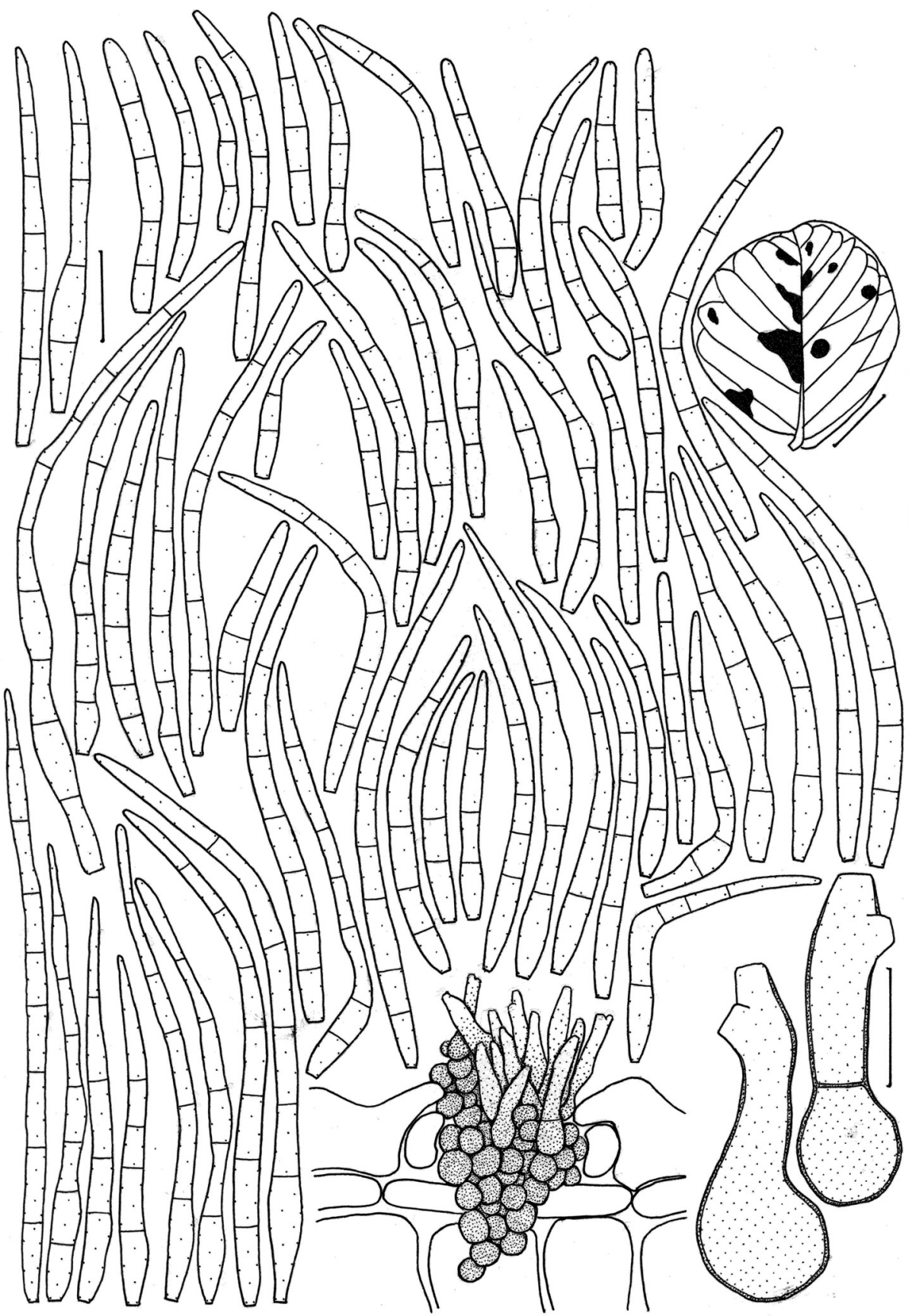

Fig. 6. Pseudocercospora stryphnodendri on Stryphnodendron adstringens. Lesions on a leaf (top right) (scale $=10 \mathrm{~mm}$ ); stomatal poorly developed stroma with a lax fascicle of short 1-septate conidiophores $($ scale $=10 \mu \mathrm{m})$; a representative sample of conidia $($ scale $=10 \mu \mathrm{m})$; a detailed view of two conidiophores, one reduced to a poliblastic conidiogenous cell (scale $=5 \mu \mathrm{m})$. Based on the holotype UB - Mycol. Col. 894 . 


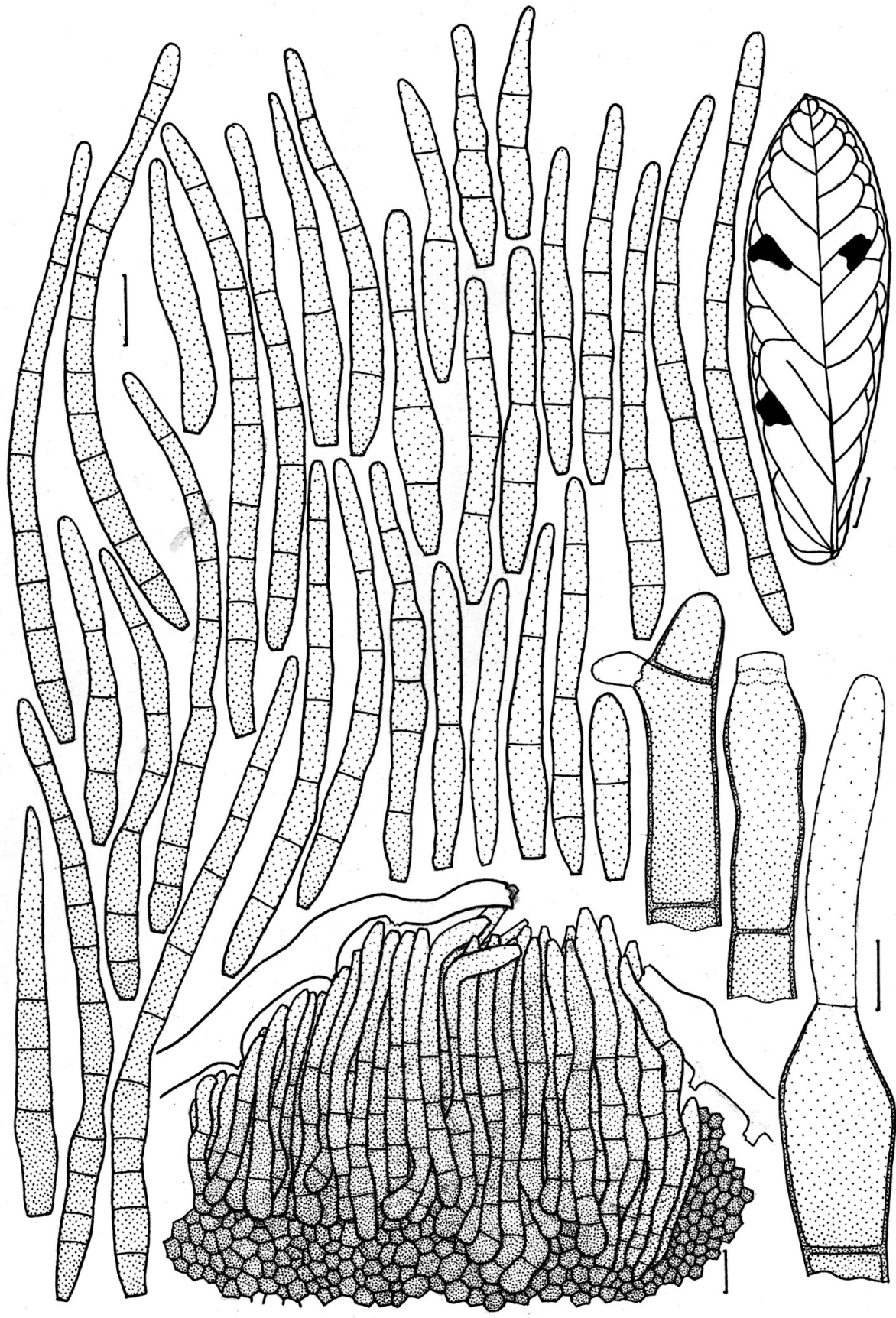

Fig. 7. Pseudocercospora subcuticularis on Senna rugosa. Lesions on a leaf (top right) (scale = $10 \mathrm{~mm}$ ); erumpent-subepidermal stroma with a compact fascicle of conidiophores (scale = $10 \mu \mathrm{m})$; a representative sample of conidia (scale $=10 \mu \mathrm{m}$ ); a detailed view of three conidiogenous cells (bottom right) $($ scale $=5 \mu \mathrm{m})$. Based on the holotype UB - Mycol. Col. 4092. 


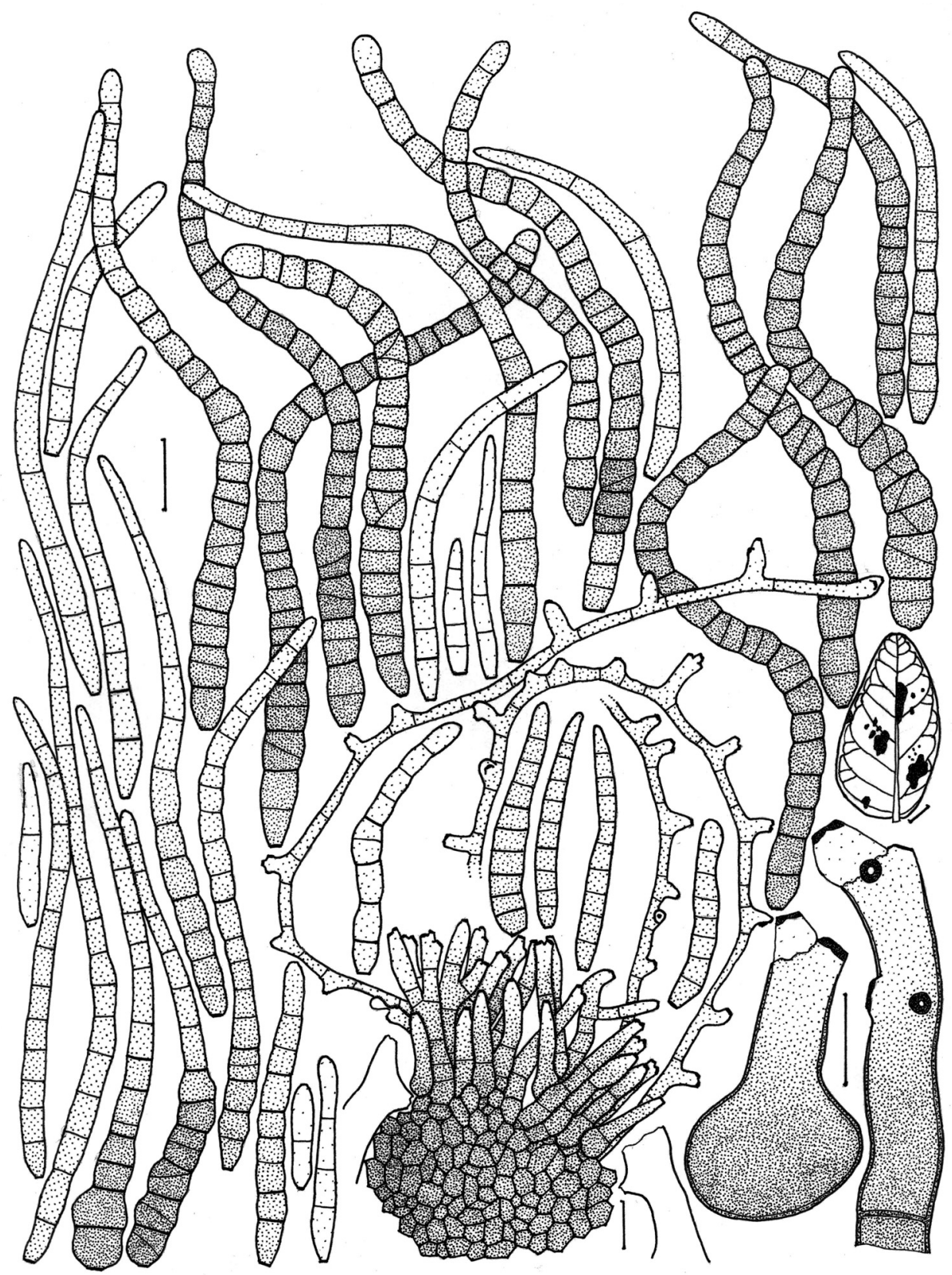

Fig. 8. Sirosporium sclerolobii on Sclerolobium paniculatum. Lesions on a leaf (scale $=10$ $\mathrm{mm}$ ); erumpent-subepidermal stroma with a conidiophore fascicle, also giving rise to fertile external hyphae $($ scale $=10 \mu \mathrm{m})$; a representative sample of polymorphic conidia (scale $=10$ $\mu \mathrm{m})$; a detailed view of two polyblastic conidiogenous cells bearing dark thickened conidiogenous loci $($ scale $=5 \mu \mathrm{m}$ ). Based on the holotype UB - Mycol. Col. 4933. 


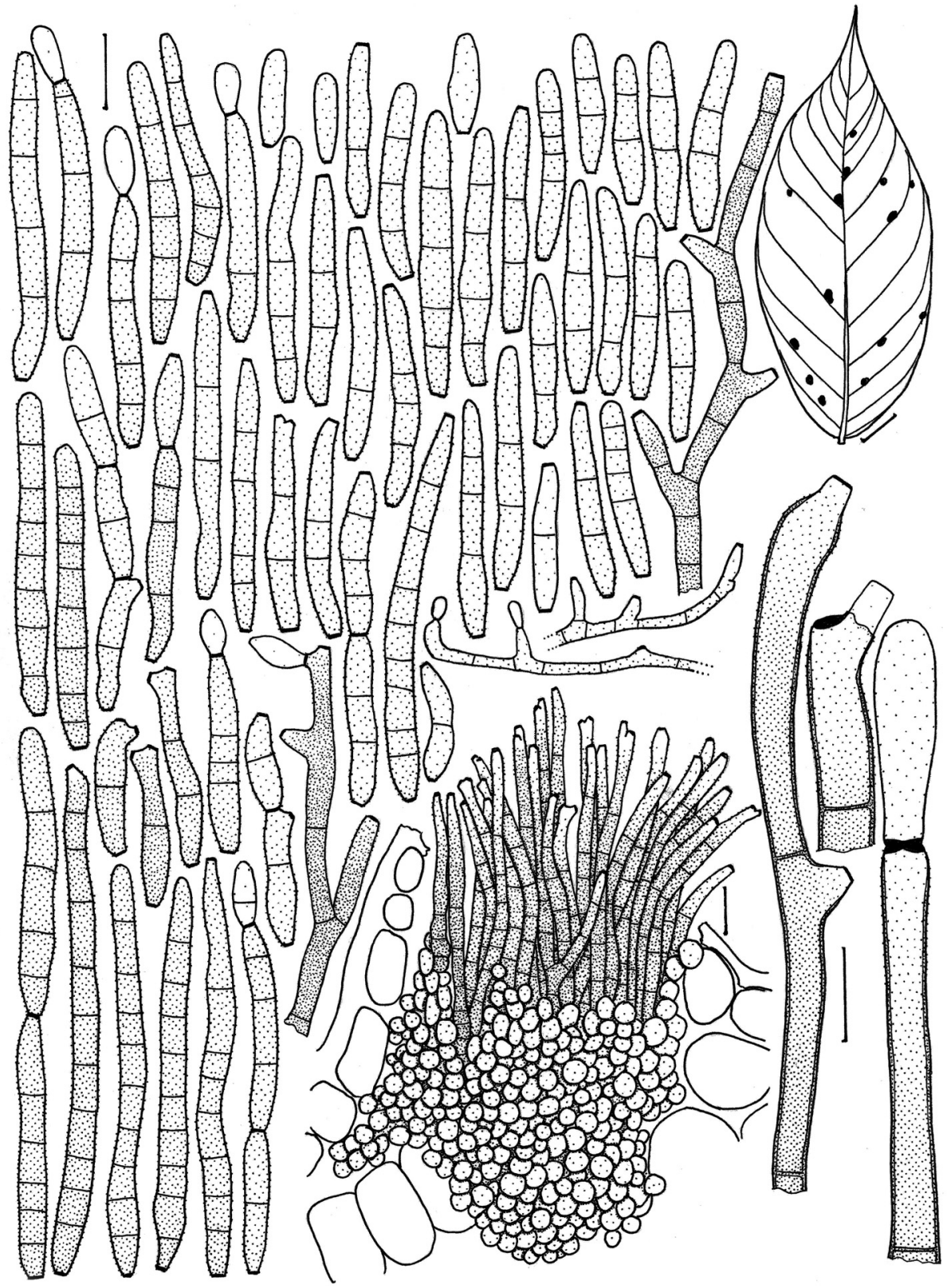

Fig. 9. Zasmidium sclerolobii on Sclerolobium paniculatum. Lesions on a leaf (top right) $($ scale $=10 \mathrm{~mm})$; erumpent-subepidermal stroma with a conidiophore fascicle $($ scale $=10$ $\mu \mathrm{m})$; a representative sample of conidia and fertile external hyphae (scale $=10 \mu \mathrm{m})$; a detailed view of three conidiogenous cells $($ scale $=5 \mu \mathrm{m}$ ). Based on the holotype UB - Mycol. Col. 6838 . 